\title{
Epidemiological Trends of Urinary Tract Infections at the Global, Regional, and National Levels from 1990-2017: A Population- Based Study
}

\section{Cong Zhu}

Wuhan University Zhongnan Hospital

\section{Qiao Huang}

Wuhan University Zhongnan Hospital

Li-Sha Luo

Wuhan University Zhongnan Hospital

\section{Tong Deng}

Wuhan University Zhongnan Hospital

\section{Jia-Min Gu}

Wuhan University Zhongnan Hospital

\section{Shi-Di Tang}

Wuhan University Zhongnan Hospital

\section{Lu-Yao Li}

Wuhan University Zhongnan Hospital

Hao Zi

Wuhan University Zhongnan Hospital

Xian-Tao Zeng ( $\square$ zengxiantao1128@whu.edu.cn )

Wuhan University https://orcid.org/0000-0003-1262-725X

\section{Research}

Keywords: Urinary tract infections, Global burden of disease, Incidence, Mortality, Disability-adjusted life years

Posted Date: January 13th, 2021

DOI: https://doi.org/10.21203/rs.3.rs-142162/v1

License: @ (i) This work is licensed under a Creative Commons Attribution 4.0 International License. Read Full License 


\section{Abstract}

Background

Urinary tract infections (UTIs) are some of the most common infections worldwide and consume a lot of medical resources every year. However, there were a lack of available data on its incidence and disease burden. We armed to investigate incidence, mortality, and disability adjusted life-years (DALYs) of urinary tract infections (UTIs) from 1990 to 2017.

Methods

We extracted data from the Global Burden of Disease Study 2017, then calculated estimated annual percentage changes (EAPC) of age-standardized incidence rate (ASIR), age-standardized death rate (ASDR), and age-standardized DALYs rate at global, national, regional, and socio-demographic index (SDI) level.

Results

From 1990 to 2017, the globally incident cases (+52.09\%), death cases $(+140.10 \%)$, and DALYs $(+69.65 \%)$ of UTIs all increased. The ASIR, ASDR, and age-standardized DALYs rate showed upward trends with the EAPC of +0.10 ( $95 \% \mathrm{Cl}: 0.07$ to 0.12$),+0.72$ (95\% Cl: $0.65-0.78)$, and +0.06 ( $95 \% \mathrm{Cl}:-0.05$ to 0.16$)$, respectively. The ASIR decreased only in the high-middle SDI quantile $(-0.26,95 \% \mathrm{Cl}:-0.3$ to -0.23$)$. United Arab Emirates had the largest increase of DALYs (+835.04\%), but Bulgaria had the largest decrease (-80.74\%). EAPC for incidence and mortality were below 0 mainly in Europe and East Asia. In 2017, the incident cases ( +3.44 times), the deaths $(+1.31$ times), and DALYs (+1.21 times) were all higher in females than males. The incident cases were mainly concentrated in 15-49 years old; DALYs and mortality were higher in over 80 age groups.

Conclusions

Globally, the burden of UTIs increased from 1990 to 2017, especially in females; however, distinct varies were observed in different regions and countries. The infants and elders are easier to die when they suffer from UTIs.

\section{Background}

Urinary tract infections (UTIs) are defined as infections that occur in any part of the urinary tract and most resulted by bacteria(1). To explore the epidemiological characteristics in different countries, ages, and gender is important to make reasonable health care policies for prevention and treatment; however, different studies presented various results. A female's lifetime risk of UTIs was estimated at $60.4 \%$ based on her self-reported doctor's diagnosis(2); another study showed that about $25 \%$ of females who have their first bacterial cystitis episode would go on to have recurrent UTIs within 6 months(3). Previous studies reported that the prevalence of UTIs was about 7.0\%-15.4\% in febrile infants, and were affected 2.5 times in male infants than females $(4,5)$. Moreover, a retrospective observational study involved almost one million patients aged $\geq 65$ years estimated incidence of clinically diagnosed UTIs in females, which showed that incidence presented an increase trend from 9.03 to 19.80 cases per 100 person-years in those over 65 years (6). Due to the high incidence of UTIs, it is estimated that UTIs cause approximately 8.6 million medical visits and 23 billion dollars in medical expenses each year $(7,8)$. Therefore, we aimed to estimate the global burden of UTIs based on the Global Burden of Disease Study (GBD) 2017, to reveal the comprehensive epidemiological characteristics of UTIs in different levels from multiple aspects.

\section{Materials And Methods}

\section{Data sources}

This study used data from the GBD 2017, which includes incidence, mortality, disability adjusted life-years (DALY) and age standardized rates (ASR) of 354 diseases and injuries of different genders and ages in 195 countries and regions around the world. Data of the incidence, death, DALYs as well as the corresponding ASR and 95\% uncertainty interval [UI] of UTIs were obtained from the Global Health Date Exchange GBD Results Tool (http://ghdx.healthdata.org/). Data processing and disease model have already been described in previous studies(9). Detailed descriptions of the methods are presented in the Methods Supplementary. 


\section{Definition of UTIs}

UTIs is defined as a kidney infection that can lead to systemic symptoms such as fever and weakness and can cause discomfort and difficulty with daily activities(9). ICD (The International Classification of Diseases ) codes include N10, N10.0, N10.9, N11, N11.0, N11.1, N11.8, N11.9, N12, N12.0, and N12.9. More detailed information on methodology of GBD 2017 can be found in the GBD studies $(10,11)$.

\section{Statistical analyses}

The ASR and their estimated annual percentage changes (EAPC) were calculated to evaluate the incidence and mortality trends of UTIs. It is shown that when EAPC and the lower boundary of the $\mathrm{Cl}$ are positive, then ASR is in an upward trend. Conversely, when EAPC and the upper boundary of the $\mathrm{Cl}$ are negative, the ASR is in a descending trend. In addition, we estimated the correlation of the EAPC to the ASR in 1990 and to the SDI in 2017.

Besides, we also analyzed the incidence, mortality, and DALYs at national, regional, and socio-demographic index (SDI) level. The age was classified into five subgroups: under $5,5-14,15-49,50-69$, and over 70 . When comparing the incidence rates of different age groups, we subdivided the age into 18 subgroups for each 5 years old from under 1 to over 80 .

All statistical analyses were performed using the $\mathrm{R}$ software (Version 3.6.1). A $p$ value less than 0.05 indicating a significant difference.

\section{Results}

\section{Incidence of Urinary Tract Infections}

Globally, incident cases of UTIs increased from 180.57 million in 1990 to 274.63 million in 2017, with an increase of $52.09 \%$ (Table 1). The age standardized incidence rate (ASIR) showed an upward trend with an EAPC of +0.10 (95\%Cl: 0.07 to 0.12 ), increasing from 3435.10 (95\%Ul: 3101.39 to 3802.41 ) to 3517.01 (95\%Ul: 3169.61 to 3884.07 ) per 100,000 persons. In 2017, the incident cases of UTIs were 212.78 million in females, which were 3.44 times higher than males (61.85 million). 
Table 1

The incidence of urinary tract infection in 1990 and 2017, and their temporal trends from 1990 to 2017.

\begin{tabular}{|c|c|c|c|c|c|c|}
\hline \multirow[t]{3}{*}{ Characteristics } & \multicolumn{2}{|l|}{1990} & \multicolumn{2}{|l|}{2017} & \multicolumn{2}{|c|}{ 1990-2017 } \\
\hline & \multirow{2}{*}{$\begin{array}{l}\text { Incident cases } \\
\text { No.×10^5(95\%UI) }\end{array}$} & \multirow{2}{*}{$\begin{array}{l}\text { ASIR per } 100,000 \\
\text { No. }(95 \% \text { UI) }\end{array}$} & \multirow{2}{*}{$\begin{array}{l}\text { Incident cases } \\
\text { No.×10^5(95\%UI) }\end{array}$} & \multirow{2}{*}{$\begin{array}{l}\text { ASIR per } 100,000 \\
\text { No. (95\%UI) }\end{array}$} & \multirow{2}{*}{$\begin{array}{l}\text { Change } \\
\text { in } \\
\text { Incidence } \\
\text { Number } \\
\text { No. (\%) }\end{array}$} & \multirow{2}{*}{$\begin{array}{l}\text { EAPC } \\
(95 \% \mathrm{Cl})\end{array}$} \\
\hline & & & & & & \\
\hline Global & $\begin{array}{l}\text { 1805.67(1623.75- } \\
2001.71)\end{array}$ & $\begin{array}{l}3435.10(3101.39- \\
3802.41)\end{array}$ & $\begin{array}{l}2746.26(2475.59- \\
3034.27)\end{array}$ & $\begin{array}{l}3517.01(3169.61- \\
3884.07)\end{array}$ & 52.09 & $\begin{array}{l}0.10(0.07- \\
0.12)\end{array}$ \\
\hline \multicolumn{7}{|l|}{ Sex } \\
\hline Male & $\begin{array}{l}418.63(377.78- \\
464.07)\end{array}$ & $\begin{array}{l}\text { 1619.52(1472.37- } \\
1783.85)\end{array}$ & $\begin{array}{l}\text { 618.46(557.65- } \\
683.47)\end{array}$ & $\begin{array}{l}\text { 1606.48(1453.16- } \\
1773.62)\end{array}$ & 47.73 & $\begin{array}{l}-0.07(-0.13 \\
\text { to }-0.01)\end{array}$ \\
\hline Female & $\begin{array}{l}1387.02(1243.39- \\
1540.25)\end{array}$ & $\begin{array}{l}5265.07(4744.66- \\
5827.55)\end{array}$ & $\begin{array}{l}2127.80(1919.74- \\
2347.15)\end{array}$ & $\begin{array}{l}5430.01(4886.07- \\
6008.72)\end{array}$ & 53.41 & $\begin{array}{l}0.13(0.09- \\
0.17)\end{array}$ \\
\hline \multicolumn{7}{|c|}{ Socio-demographic index } \\
\hline Low SDI & $\begin{array}{l}\text { 181.21(162.20- } \\
202.58)\end{array}$ & $\begin{array}{l}2835.38(2561.56- \\
3146.24)\end{array}$ & $\begin{array}{l}360.54(321.15- \\
403.36)\end{array}$ & $\begin{array}{l}3004.74(2682.66- \\
3346.15)\end{array}$ & 98.96 & $\begin{array}{l}0.20(0.14- \\
0.26)\end{array}$ \\
\hline $\begin{array}{l}\text { Low-middle } \\
\text { SDI }\end{array}$ & $\begin{array}{l}302.59(270.06- \\
338.58)\end{array}$ & $\begin{array}{l}3064.20(2752.78- \\
3405.10)\end{array}$ & $\begin{array}{l}559.95(498.41- \\
624.22)\end{array}$ & $\begin{array}{l}3367.50(3010.09- \\
3748.65)\end{array}$ & 85.05 & $\begin{array}{l}0.37(0.36- \\
0.38)\end{array}$ \\
\hline Middle SDI & $\begin{array}{l}463.40(412.82- \\
518.68)\end{array}$ & $\begin{array}{l}3075.72(2761.99- \\
3414.76)\end{array}$ & $\begin{array}{l}736.76(658.18- \\
823.36)\end{array}$ & $\begin{array}{l}3353.54(2997.57- \\
3733.36)\end{array}$ & 58.99 & $\begin{array}{l}0.27(0.24- \\
0.31)\end{array}$ \\
\hline $\begin{array}{l}\text { High-middle } \\
\text { SDI }\end{array}$ & $\begin{array}{l}434.63(390.54- \\
482.23)\end{array}$ & $\begin{array}{l}3864.51(3478.93- \\
4281.97)\end{array}$ & $\begin{array}{l}550.09(492.25- \\
613.62)\end{array}$ & $\begin{array}{l}3674.53(3292.02- \\
4084.40)\end{array}$ & 26.56 & $\begin{array}{l}-0.26(-0.30 \\
\text { to }-0.23)\end{array}$ \\
\hline High SDI & $\begin{array}{l}416.88(375.61- \\
461.10)\end{array}$ & $\begin{array}{l}4151.87(3750.68- \\
4586.38)\end{array}$ & $\begin{array}{l}529.15(497.59- \\
564.36)\end{array}$ & $\begin{array}{l}4231.47(3949.74- \\
4537.56)\end{array}$ & 26.93 & $\begin{array}{l}0.10(0.07- \\
0.13)\end{array}$ \\
\hline \multicolumn{7}{|l|}{ Region } \\
\hline $\begin{array}{l}\text { Andean Latin } \\
\text { America }\end{array}$ & $\begin{array}{l}18.31(16.29- \\
20.52)\end{array}$ & $\begin{array}{l}4941.72(4422.17- \\
5514.40)\end{array}$ & $\begin{array}{l}32.53(28.87- \\
36.44)\end{array}$ & $\begin{array}{l}5262.60(4682.67- \\
5889.21)\end{array}$ & 77.68 & $\begin{array}{l}0.30(0.27- \\
0.32)\end{array}$ \\
\hline Australasia & $\begin{array}{l}10.47(9.26- \\
11.83)\end{array}$ & $\begin{array}{l}5007.24(4427.70- \\
5657.87)\end{array}$ & $\begin{array}{l}15.18(13.60- \\
16.98)\end{array}$ & $\begin{array}{l}5302.77(4709.26- \\
5951.26)\end{array}$ & 44.97 & $\begin{array}{l}0.22(0.21- \\
0.24)\end{array}$ \\
\hline Caribbean & $\begin{array}{l}13.34(11.81- \\
14.98)\end{array}$ & $\begin{array}{l}3751.06(3341.45- \\
4189.53)\end{array}$ & $\begin{array}{l}19.00(16.84- \\
21.18)\end{array}$ & $\begin{array}{l}4039.03(3590.53- \\
4500.18)\end{array}$ & 42.48 & $\begin{array}{l}0.28(0.27- \\
0.29)\end{array}$ \\
\hline Central Asia & $\begin{array}{l}36.72(32.74- \\
41.26)\end{array}$ & $\begin{array}{l}5265.72(4716.08- \\
5875.25)\end{array}$ & $\begin{array}{l}48.18(42.84- \\
54.19)\end{array}$ & $\begin{array}{l}5128.76(4580.77- \\
5746.59)\end{array}$ & 31.22 & $\begin{array}{l}-0.02(-0.10 \\
\text { to } 0.05)\end{array}$ \\
\hline Central Europe & $\begin{array}{l}52.67(47.64- \\
58.12)\end{array}$ & $\begin{array}{l}4284.89(3856.31- \\
4746.60)\end{array}$ & $\begin{array}{l}45.56(41.21- \\
50.25)\end{array}$ & $\begin{array}{l}4126.03(3694.61- \\
4581.07)\end{array}$ & -13.50 & $\begin{array}{l}-0.12(-0.21 \\
\text { to }-0.03)\end{array}$ \\
\hline $\begin{array}{l}\text { Central Latin } \\
\text { America }\end{array}$ & $\begin{array}{l}41.81(37.32- \\
46.74)\end{array}$ & $\begin{array}{l}2714.41(2440.61- \\
3017.87)\end{array}$ & $\begin{array}{l}71.38(63.85- \\
79.47)\end{array}$ & $\begin{array}{l}2787.89(2497.98- \\
3094.04)\end{array}$ & 70.70 & $\begin{array}{l}0.13(0.07- \\
0.20)\end{array}$ \\
\hline $\begin{array}{l}\text { Central Sub- } \\
\text { Saharan } \\
\text { Africa }\end{array}$ & $8.71(7.65-9.87)$ & $\begin{array}{l}\text { 1701.64(1509.23- } \\
1903.02)\end{array}$ & $\begin{array}{l}20.63(18.19- \\
23.38)\end{array}$ & $\begin{array}{l}\text { 1808.50(1599.84- } \\
2023.63)\end{array}$ & 136.83 & $\begin{array}{l}0.24(0.18- \\
0.30)\end{array}$ \\
\hline East Asia & $\begin{array}{l}361.37(320.39- \\
402.94)\end{array}$ & $\begin{array}{l}2914.88(2604.08- \\
3244.79)\end{array}$ & $\begin{array}{l}512.06(453.32- \\
574.68)\end{array}$ & $\begin{array}{l}2967.33(2655.80- \\
3299.94)\end{array}$ & 41.70 & $\begin{array}{l}-0.13(-0.24 \\
\text { to }-0.03)\end{array}$ \\
\hline $\begin{array}{l}\text { Eastern } \\
\text { Europe }\end{array}$ & $\begin{array}{l}152.24(137.31- \\
168.52)\end{array}$ & $\begin{array}{l}6708.99(6047.89- \\
7455.44)\end{array}$ & $\begin{array}{l}127.77(114.01- \\
142.20)\end{array}$ & $\begin{array}{l}6185.69(5548.09- \\
6900.51)\end{array}$ & -16.07 & $\begin{array}{l}-0.35(-0.42 \\
\text { to }-0.28)\end{array}$ \\
\hline
\end{tabular}

ASIR: age standardized incidence rate; EAPC: estimated annual percentage change; Cl: confidence interval; UI: uncertainty interval; SDI: socio-demographic index 


\begin{tabular}{|c|c|c|c|c|c|c|}
\hline \multirow[t]{3}{*}{ Characteristics } & \multicolumn{2}{|l|}{1990} & \multicolumn{2}{|l|}{2017} & \multicolumn{2}{|c|}{ 1990-2017 } \\
\hline & \multirow{2}{*}{$\begin{array}{l}\text { Incident cases } \\
\text { No.×10^5(95\%UI) }\end{array}$} & \multirow{2}{*}{$\begin{array}{l}\text { ASIR per } 100,000 \\
\text { No. (95\%UI) }\end{array}$} & \multirow{2}{*}{$\begin{array}{l}\text { Incident cases } \\
\text { No.×10^5(95\%UI) }\end{array}$} & \multirow{2}{*}{$\begin{array}{l}\text { ASIR per } 100,000 \\
\text { No. }(95 \% \text { UI) }\end{array}$} & \multirow{2}{*}{$\begin{array}{l}\text { Change } \\
\text { in } \\
\text { Incidence } \\
\text { Number } \\
\text { No. (\%) }\end{array}$} & \multirow{2}{*}{$\begin{array}{l}\text { EAPC } \\
(95 \% \mathrm{Cl})\end{array}$} \\
\hline & & & & & & \\
\hline $\begin{array}{l}\text { Eastern Sub- } \\
\text { Saharan } \\
\text { Africa }\end{array}$ & $\begin{array}{l}36.36(32.29- \\
40.91)\end{array}$ & $\begin{array}{l}2122.76(1912.08- \\
2357.52)\end{array}$ & $\begin{array}{l}77.49(68.62- \\
87.31)\end{array}$ & $\begin{array}{l}2178.53(1949.35- \\
2426.55)\end{array}$ & 113.15 & $\begin{array}{l}0.06(0.010- \\
0.11)\end{array}$ \\
\hline $\begin{array}{l}\text { High-income } \\
\text { Asia Pacific }\end{array}$ & $\begin{array}{l}\text { 91.27(81.75- } \\
101.31)\end{array}$ & $\begin{array}{l}5572.61(5013.78- \\
6196.22)\end{array}$ & $\begin{array}{l}93.43(85.20- \\
102.70)\end{array}$ & $\begin{array}{l}5769.18(5209.47- \\
6390.64)\end{array}$ & 2.37 & $\begin{array}{l}0.21(0.18- \\
0.25)\end{array}$ \\
\hline $\begin{array}{l}\text { High-income } \\
\text { North America }\end{array}$ & $\begin{array}{l}174.13(157.95- \\
192.12)\end{array}$ & $\begin{array}{l}5664.12(5122.82- \\
6238.00)\end{array}$ & $\begin{array}{l}245.99(237.58- \\
254.68)\end{array}$ & $\begin{array}{l}5607.41(5414.55- \\
5811.25)\end{array}$ & 41.27 & $\begin{array}{l}0.09(0.04- \\
0.14)\end{array}$ \\
\hline $\begin{array}{l}\text { North Africa } \\
\text { and Middle } \\
\text { East }\end{array}$ & $\begin{array}{l}79.81(70.93- \\
89.26)\end{array}$ & $\begin{array}{l}2428.43(2159.62- \\
2708.20)\end{array}$ & $\begin{array}{l}153.26(135.11- \\
171.59)\end{array}$ & $\begin{array}{l}2492.61(2218.48- \\
2780.83)\end{array}$ & 92.03 & $\begin{array}{l}0.10(0.09- \\
0.11)\end{array}$ \\
\hline Oceania & $2.17(1.95-2.40)$ & $\begin{array}{l}3631.22(3299.29- \\
4014.03)\end{array}$ & $4.47(3.97-4.97)$ & $\begin{array}{l}3733.35(3346.62- \\
4149.60)\end{array}$ & 106.02 & $\begin{array}{l}0.03(0.00- \\
0.06)\end{array}$ \\
\hline South Asia & $\begin{array}{l}341.12(304.77- \\
382.85)\end{array}$ & $\begin{array}{l}3199.37(2875.30- \\
3561.61)\end{array}$ & $\begin{array}{l}661.82(587.4- \\
740.52)\end{array}$ & $\begin{array}{l}3691.52(3297.58- \\
4112.62)\end{array}$ & 94.01 & $\begin{array}{l}0.54(0.50- \\
0.58)\end{array}$ \\
\hline $\begin{array}{l}\text { Southeast } \\
\text { Asia }\end{array}$ & $\begin{array}{l}126.11(112.88- \\
140.71)\end{array}$ & $\begin{array}{l}2908.19(2616.16- \\
3224.24)\end{array}$ & $\begin{array}{l}206.88(184.56- \\
231.48)\end{array}$ & $\begin{array}{l}3057.58(2738.56- \\
3402.25)\end{array}$ & 64.04 & $\begin{array}{l}0.09(0.00- \\
0.18)\end{array}$ \\
\hline $\begin{array}{l}\text { Southern Latin } \\
\text { America }\end{array}$ & $\begin{array}{l}18.97(16.95- \\
21.07)\end{array}$ & $\begin{array}{l}3868.66(3464.00- \\
4297.91)\end{array}$ & $\begin{array}{l}30.04(27.13- \\
33.26)\end{array}$ & $\begin{array}{l}4338.49(3901.56- \\
4828.94)\end{array}$ & 58.37 & $\begin{array}{l}0.43(0.4- \\
0.46)\end{array}$ \\
\hline $\begin{array}{l}\text { Southern Sub- } \\
\text { Saharan } \\
\text { Africa }\end{array}$ & $\begin{array}{l}11.06(9.73- \\
12.50)\end{array}$ & $\begin{array}{l}2176.57(1935.09- \\
2442.13)\end{array}$ & $\begin{array}{l}17.86(15.67- \\
20.26)\end{array}$ & $\begin{array}{l}2253.41(1988.98- \\
2524.25)\end{array}$ & 61.55 & $\begin{array}{l}0.2(0.00- \\
0.40)\end{array}$ \\
\hline $\begin{array}{l}\text { Tropical Latin } \\
\text { America }\end{array}$ & $\begin{array}{l}\text { 91.89(81.22-- } \\
103.92)\end{array}$ & $\begin{array}{l}6107.45(5429.33- \\
6854.30)\end{array}$ & $\begin{array}{l}\text { 161.58(142.2- } \\
181.99)\end{array}$ & $\begin{array}{l}7050.31(6231.68- \\
7918.56)\end{array}$ & 75.84 & $\begin{array}{l}0.67(0.58- \\
0.76)\end{array}$ \\
\hline $\begin{array}{l}\text { Western } \\
\text { Europe }\end{array}$ & $\begin{array}{l}94.75(82.18- \\
108.77)\end{array}$ & $\begin{array}{l}2372.67(2054.69- \\
2733.09)\end{array}$ & $\begin{array}{l}107.28(94.13- \\
121.73)\end{array}$ & $\begin{array}{l}2592.19(2255.78- \\
2973.83)\end{array}$ & 13.23 & $\begin{array}{l}0.43(0.37- \\
0.49)\end{array}$ \\
\hline $\begin{array}{l}\text { Western Sub- } \\
\text { Saharan } \\
\text { Africa }\end{array}$ & $\begin{array}{l}42.39(37.95- \\
47.50)\end{array}$ & $\begin{array}{l}2422.05(2185.69- \\
2688.29)\end{array}$ & $\begin{array}{l}93.85(83.33- \\
105.07)\end{array}$ & $\begin{array}{l}2391.08(2137.03- \\
2667.75)\end{array}$ & 121.38 & $\begin{array}{l}-0.07(-0.11 \\
\text { to }-0.04)\end{array}$ \\
\hline
\end{tabular}

ASIR: age standardized incidence rate; EAPC: estimated annual percentage change; Cl: confidence interval; UI: uncertainty interval; SDI: socio-demographic index

The ASIR of UTIs increased in most SDI quintiles except for the high-middle SDI quantile(Fig. 1A). The greatest increase of ASIR was observed in Tropical Latin America (EAPC $=+0.67,95 \% \mathrm{Cl}$ : 0.58 to 0.76 ) (Fig. 2A). The country with highest ASIR was New Zealand (8289.60 per 100,000 persons) in 2017 (Fig. 3A). The incidence of UTIs in 195 countries and territories were detailed in Supplementary Table 1.

In addition, there was no significant association between $\operatorname{EAPC}$ and $\operatorname{SDI}(\rho=0.03, p=0.684)$, but ASIR showed a positive correlation with SDI (Fig. 4A) and the EAPC was negatively correlated with the relative ASIR in $1990(\rho=-0.23, p<0.001$, Fig. 5A).

Death from Urinary Tract Infections

Globally, the death cases of UTIs were 206,388 in 2017, which increased $140.10 \%$ from 1990. Age-standardized death rate (ASDR) increased from 2.25 (95\% Ul: $2.08-2.46)$ to 2.74 (95\%Ul: 2.63-2.96) per 100,000 persons with an EAPC of +0.72 (95\% Cl: $0.65-0.78)$ (Table 2). 
Table 2

The deaths of urinary tract infection in 1990 and 2017, and their temporal trends from 1990 to 2017.

\begin{tabular}{|c|c|c|c|c|c|c|}
\hline \multirow[t]{4}{*}{ Characteristics } & \multicolumn{2}{|l|}{1990} & \multicolumn{2}{|l|}{2017} & \multicolumn{2}{|c|}{ 1990-2017 } \\
\hline & Deaths & $\begin{array}{l}\text { ASDR per } \\
100,000\end{array}$ & Deaths & $\begin{array}{l}\text { ASDR per } \\
100,000\end{array}$ & Change in & $\begin{array}{l}\text { EAPC No. } \\
(95 \% \mathrm{Cl})\end{array}$ \\
\hline & \multirow[t]{2}{*}{$\begin{array}{l}\text { No. } \times 10^{3}(95 \% \\
\text { Ul) }\end{array}$} & \multirow[t]{2}{*}{ No. (95\% UI) } & \multirow[t]{2}{*}{$\begin{array}{l}\text { No. } \times 10^{3}(95 \% \\
\text { UI) }\end{array}$} & No. (95\% UI) & \multirow{2}{*}{\multicolumn{2}{|c|}{$\begin{array}{l}\text { Death } \\
\text { Number } \\
\text { No. (\%) }\end{array}$}} \\
\hline & & & & & & \\
\hline Global & $\begin{array}{l}85.96(77.84- \\
94.85)\end{array}$ & $\begin{array}{l}2.25(2.08- \\
2.46)\end{array}$ & $\begin{array}{l}206.39(197.90- \\
223.16)\end{array}$ & $\begin{array}{l}2.74(2.63- \\
2.96)\end{array}$ & 140.10 & $\begin{array}{l}0.72(0.65- \\
0.78)\end{array}$ \\
\hline \multicolumn{7}{|l|}{ Sex } \\
\hline male & $\begin{array}{l}40.66(35.70- \\
45.61)\end{array}$ & $\begin{array}{l}2.51(2.26- \\
2.76)\end{array}$ & $\begin{array}{l}89.82(84.18- \\
99.56)\end{array}$ & $\begin{array}{l}2.76(2.60- \\
3.03)\end{array}$ & 120.93 & $\begin{array}{l}0.38(0.31- \\
0.45)\end{array}$ \\
\hline female & $\begin{array}{l}45.30(40.72- \\
50.55)\end{array}$ & $\begin{array}{l}2.12(1.93- \\
2.34)\end{array}$ & $\begin{array}{l}116.57(111.27- \\
125.67)\end{array}$ & $\begin{array}{l}2.74(2.62- \\
2.96)\end{array}$ & 157.31 & $\begin{array}{l}0.92(0.83- \\
1.00)\end{array}$ \\
\hline \multicolumn{7}{|c|}{ Socio-demographic index } \\
\hline Low SDI & $\begin{array}{l}13.20(9.56- \\
18.23)\end{array}$ & $\begin{array}{l}3.82(3.03- \\
5.18)\end{array}$ & $\begin{array}{l}29.24(25.62- \\
36.27)\end{array}$ & $\begin{array}{l}4.64(4.12- \\
5.70)\end{array}$ & 121.49 & $\begin{array}{l}0.59(0.36- \\
0.82)\end{array}$ \\
\hline Low-middle SDI & $\begin{array}{l}\text { 16.94(14.24- } \\
19.61)\end{array}$ & $\begin{array}{l}2.94(2.54- \\
3.36)\end{array}$ & $\begin{array}{l}43.51(39.8- \\
48.94)\end{array}$ & $\begin{array}{l}4.09(3.74- \\
4.55)\end{array}$ & 156.84 & $\begin{array}{l}1.13(1.01- \\
1.25)\end{array}$ \\
\hline Middle SDI & $\begin{array}{l}16.91(14.79- \\
18.37)\end{array}$ & $\begin{array}{l}1.87(1.67- \\
2.03)\end{array}$ & $\begin{array}{l}45.67(43.32- \\
48.94)\end{array}$ & $\begin{array}{l}2.33(2.22- \\
2.51)\end{array}$ & 170.08 & $\begin{array}{l}0.67(0.52- \\
0.81)\end{array}$ \\
\hline High-middle SDI & $\begin{array}{l}16.06(14.68- \\
17.01)\end{array}$ & $\begin{array}{l}1.82(1.67- \\
1.92)\end{array}$ & $\begin{array}{l}32.43(31.01- \\
34.03)\end{array}$ & $\begin{array}{l}2.02(1.93- \\
2.11)\end{array}$ & 101.91 & $\begin{array}{l}0.18(-0.06 \text { to } \\
0.42)\end{array}$ \\
\hline High SDI & $\begin{array}{l}22.67(22.07- \\
23.93)\end{array}$ & $\begin{array}{l}1.78(1.74- \\
1.88)\end{array}$ & $\begin{array}{l}55.26(53.33- \\
56.60)\end{array}$ & $\begin{array}{l}2.06(1.98- \\
2.11)\end{array}$ & 143.75 & $\begin{array}{l}0.85(0.69- \\
1.00)\end{array}$ \\
\hline \multicolumn{7}{|l|}{ Region } \\
\hline Andean Latin America & $\begin{array}{l}0.86(0.79- \\
0.93)\end{array}$ & $\begin{array}{l}3.47(3.24- \\
3.71)\end{array}$ & $1.79(1.63-1.98)$ & $\begin{array}{l}3.32(3.01- \\
3.67)\end{array}$ & 107.89 & $\begin{array}{l}-0.35(-0.67 \text { to } \\
-0.02)\end{array}$ \\
\hline Australasia & $\begin{array}{l}0.32(0.30- \\
0.35)\end{array}$ & $\begin{array}{l}1.38(1.30- \\
1.52)\end{array}$ & $0.99(0.90-1.09)$ & $\begin{array}{l}1.67(1.52- \\
1.83)\end{array}$ & 214.52 & $\begin{array}{l}0.85(0.53- \\
1.18)\end{array}$ \\
\hline Caribbean & $\begin{array}{l}0.44(0.40- \\
0.49)\end{array}$ & $\begin{array}{l}1.61(1.49- \\
1.76)\end{array}$ & $1.24(1.16-1.34)$ & $\begin{array}{l}2.49(2.31- \\
2.68)\end{array}$ & 181.18 & $\begin{array}{l}1.73(1.58- \\
1.87)\end{array}$ \\
\hline Central Asia & $\begin{array}{l}1.38(1.30- \\
1.43)\end{array}$ & $\begin{array}{l}2.53(2.37- \\
2.64)\end{array}$ & $2.94(2.78-3.11)$ & $\begin{array}{l}3.82(3.59- \\
4.03)\end{array}$ & 113.80 & $\begin{array}{l}1.38(1.16- \\
1.61)\end{array}$ \\
\hline Central Europe & $\begin{array}{l}3.73(3.47- \\
3.87)\end{array}$ & $\begin{array}{l}2.58(2.4- \\
2.67)\end{array}$ & $3.09(2.97-3.25)$ & $\begin{array}{l}1.40(1.35- \\
1.47)\end{array}$ & -17.32 & $\begin{array}{l}-2.13(-3.06 \text { to } \\
-1.2)\end{array}$ \\
\hline Central Latin America & $\begin{array}{l}2.41(2.31- \\
2.56)\end{array}$ & $\begin{array}{l}2.77(2.66- \\
2.97)\end{array}$ & $8.30(7.73-8.66)$ & $\begin{array}{l}3.70(3.45- \\
3.86)\end{array}$ & 244.62 & $\begin{array}{l}1.47(1.07- \\
1.87)\end{array}$ \\
\hline $\begin{array}{l}\text { Central Sub-Saharan } \\
\text { Africa }\end{array}$ & $\begin{array}{l}0.36(0.22- \\
0.69)\end{array}$ & $\begin{array}{l}1.48(0.97- \\
2.49)\end{array}$ & $0.80(0.56-1.36)$ & $\begin{array}{l}1.59(1.08- \\
2.59)\end{array}$ & 122.70 & $\begin{array}{l}0.00(-0.23 \text { to } \\
0.22)\end{array}$ \\
\hline East Asia & $\begin{array}{l}7.94(6.21- \\
9.07)\end{array}$ & $\begin{array}{l}0.94(0.75- \\
1.07)\end{array}$ & $\begin{array}{l}10.11(9.46- \\
11.24)\end{array}$ & $\begin{array}{l}0.59(0.56- \\
0.66)\end{array}$ & 27.38 & $\begin{array}{l}-2.29(-2.53 \text { to } \\
-2.05)\end{array}$ \\
\hline Eastern Europe & $\begin{array}{l}7.52(6.54- \\
8.08)\end{array}$ & $\begin{array}{l}2.71(2.37- \\
2.91)\end{array}$ & $7.96(7.48-8.22)$ & $\begin{array}{l}2.38(2.25- \\
2.47)\end{array}$ & 5.87 & $\begin{array}{l}-1.34(-1.74 \text { to } \\
-0.94)\end{array}$ \\
\hline $\begin{array}{l}\text { Eastern Sub-Saharan } \\
\text { Africa }\end{array}$ & $\begin{array}{l}2.77(1.57- \\
5.41)\end{array}$ & $\begin{array}{l}3.56(2.22- \\
6.35)\end{array}$ & $5.61(3.78-9.35)$ & $\begin{array}{l}3.76(2.47- \\
6.10)\end{array}$ & 102.60 & $\begin{array}{l}-0.05(-0.18 \text { to } \\
0.09)\end{array}$ \\
\hline
\end{tabular}

ASDR: age standardized death rate; EAPC: estimated annual percentage change; Cl: confidence interval; UI: uncertainty interval; SDI: socio-demographic index 


\begin{tabular}{|c|c|c|c|c|c|c|}
\hline \multirow[t]{4}{*}{ Characteristics } & \multicolumn{2}{|l|}{1990} & \multicolumn{2}{|l|}{2017} & \multicolumn{2}{|l|}{$1990-2017$} \\
\hline & Deaths & $\begin{array}{l}\text { ASDR per } \\
100,000\end{array}$ & Deaths & $\begin{array}{l}\text { ASDR per } \\
100,000\end{array}$ & Change in & $\begin{array}{l}\text { EAPC No. } \\
(95 \% \mathrm{Cl})\end{array}$ \\
\hline & \multirow[t]{2}{*}{$\begin{array}{l}\text { No. } \times 10^{3}(95 \% \\
\text { UI) }\end{array}$} & \multirow[t]{2}{*}{ No. (95\% UI) } & \multirow[t]{2}{*}{$\begin{array}{l}\text { No. } \times 10^{3}(95 \% \\
\text { Ul) }\end{array}$} & No. (95\% UI) & \multirow{2}{*}{\multicolumn{2}{|c|}{$\begin{array}{l}\text { Death } \\
\text { Number } \\
\text { No. (\%) }\end{array}$}} \\
\hline & & & & & & \\
\hline $\begin{array}{l}\text { High-income Asia } \\
\text { Pacific }\end{array}$ & $\begin{array}{l}1.76(1.70- \\
1.83)\end{array}$ & $\begin{array}{l}1.04(1.00- \\
1.07)\end{array}$ & $6.72(6.37-7.21)$ & $\begin{array}{l}1.13(1.07- \\
1.22)\end{array}$ & 281.27 & $\begin{array}{l}0.80(0.48- \\
1.11)\end{array}$ \\
\hline $\begin{array}{l}\text { High-income North } \\
\text { America }\end{array}$ & $\begin{array}{l}9.59(9.32- \\
9.86)\end{array}$ & $\begin{array}{l}2.52(2.44- \\
2.59)\end{array}$ & $\begin{array}{l}18.96(18.29- \\
19.48)\end{array}$ & $\begin{array}{l}2.75(2.64- \\
2.82)\end{array}$ & 97.66 & $\begin{array}{l}0.35(0.09- \\
0.62)\end{array}$ \\
\hline $\begin{array}{l}\text { North Africa and } \\
\text { Middle East }\end{array}$ & $\begin{array}{l}1.57(1.31- \\
1.83)\end{array}$ & $\begin{array}{l}0.81(0.71- \\
0.91)\end{array}$ & $3.81(3.60-3.99)$ & $\begin{array}{l}1.02(0.95- \\
1.07)\end{array}$ & 142.62 & $\begin{array}{l}0.81(0.60- \\
1.03)\end{array}$ \\
\hline Oceania & $\begin{array}{l}0.12(0.10- \\
0.14)\end{array}$ & $\begin{array}{l}3.94(3.45- \\
4.55)\end{array}$ & $0.22(0.19-0.26)$ & $\begin{array}{l}3.65(3.25- \\
4.12)\end{array}$ & 84.83 & $\begin{array}{l}-0.55(-0.69 \text { to } \\
-0.42)\end{array}$ \\
\hline South Asia & $\begin{array}{l}21.76(16.89- \\
26)\end{array}$ & $\begin{array}{l}3.73(3.04- \\
4.48)\end{array}$ & $\begin{array}{l}61.54(55.99- \\
68.20)\end{array}$ & $\begin{array}{l}5.35(4.87- \\
5.92)\end{array}$ & 182.82 & $\begin{array}{l}1.30(1.16- \\
1.44)\end{array}$ \\
\hline Southeast Asia & $\begin{array}{l}5.92(4.89- \\
6.70)\end{array}$ & $\begin{array}{l}2.39(1.99- \\
2.72)\end{array}$ & $\begin{array}{l}17.73(15.84- \\
21.04)\end{array}$ & $\begin{array}{l}3.35(2.97- \\
4.07)\end{array}$ & 199.64 & $\begin{array}{l}1.02(0.81- \\
1.22)\end{array}$ \\
\hline $\begin{array}{l}\text { Southern Latin } \\
\text { America }\end{array}$ & $\begin{array}{l}1.53(1.47- \\
1.60)\end{array}$ & $\begin{array}{l}3.53(3.40- \\
3.68)\end{array}$ & $5.04(4.66-5.44)$ & $\begin{array}{l}5.88(5.44- \\
6.35)\end{array}$ & 228.79 & $\begin{array}{l}2.12(1.93- \\
2.32)\end{array}$ \\
\hline $\begin{array}{l}\text { Southern Sub- } \\
\text { Saharan Africa }\end{array}$ & $\begin{array}{l}0.42(0.32- \\
0.48)\end{array}$ & $\begin{array}{l}1.37(1.06- \\
1.58)\end{array}$ & $0.93(0.83-1.15)$ & $\begin{array}{l}1.73(1.54- \\
2.15)\end{array}$ & 122.03 & $\begin{array}{l}0.74(-0.04 \text { to } \\
1.52)\end{array}$ \\
\hline Tropical Latin America & $\begin{array}{l}3.08(2.94- \\
3.20)\end{array}$ & $\begin{array}{l}3.71(3.57- \\
3.84)\end{array}$ & $\begin{array}{l}16.99(15.99- \\
17.59)\end{array}$ & $\begin{array}{l}7.84(7.39- \\
8.12)\end{array}$ & 452.08 & $\begin{array}{l}3.31(2.86- \\
3.75)\end{array}$ \\
\hline Western Europe & $\begin{array}{l}8.28(7.89- \\
9.12)\end{array}$ & $\begin{array}{l}1.37(1.31- \\
1.51)\end{array}$ & $\begin{array}{l}25.16(23.94- \\
26.08)\end{array}$ & $\begin{array}{l}2.14(2.03- \\
2.22)\end{array}$ & 203.71 & $\begin{array}{l}2.25(1.91- \\
2.60)\end{array}$ \\
\hline $\begin{array}{l}\text { Western Sub-Saharan } \\
\text { Africa }\end{array}$ & $\begin{array}{l}4.20(3.43- \\
5.11)\end{array}$ & $\begin{array}{l}4.16(3.43- \\
4.9)\end{array}$ & $6.46(5.24-8.34)$ & $\begin{array}{l}3.25(2.58- \\
4.26)\end{array}$ & 53.62 & $\begin{array}{l}-1.54(-1.85 \text { to } \\
-1.22)\end{array}$ \\
\hline
\end{tabular}

ASDR: age standardized death rate; EAPC: estimated annual percentage change; Cl: confidence interval; Ul: uncertainty interval; SDI: socio-demographic index

Regionally, the death cases of UTIs showed an increase trend in all SDI quintiles and the highest was recorded in the high SDI quintile $(55,257)$ (Table 2). ASDR of UTIs increased largest in Tropical Latin America (Fig. 2B). However, the ASDR decreased in the high SDI quintile and the high-middle SDI quintile among males (Fig. 1B). Nationally, the top three countries with the highest death cases of UTIs were India, the United States and Brazil in 2017 (Supplementary Table 2). The ASDR was highest in Seychelles (8.67 per 100,000 persons) while lowest in Macedonia (0.14 per 100,000 persons) (Fig. 6); the detailed .data recorded in Supplementary Table 3.

ASDR mostly showed a decrease trend with the SDI, but only increased at SDI values from 0.60 to 0.69 (Fig. 4B). EAPC was negatively correlated with $\operatorname{ASDR}(\rho=-0.41, p<.001)$, but not correlated with $\operatorname{SDI}(\rho=0.13, p=.07$, Fig. $5 \mathrm{~B})$.

\section{DALYs of Urinary Tract Infections}

Globally, DALYs of UTIs was 1.70 times higher in $2017(4,695,291.23)$ than $1990(2,767,666.19)$. The ASR of DALYs increased from 57.32 (95\% Ul: 49.72 to 64.50 ) in 1990 to 60.66 (95\% Ul: 57.21 to 67.20) per 100,000 persons in 2017 (Table 3). The ASR of DALYs showed an increase trend among females (EAPC $=+0.21,95 \% \mathrm{Cl}$ : 0.08 to 0.35 ), while showed a decreased trend among males $(E A P C=-0.15,95 \% \mathrm{Cl}:-0.23$ to -0.06$)$.

Table 3. The DALY of urinary tract infections, and their temporal trends from 1990 to 2017. 


\begin{tabular}{|c|c|c|c|c|c|c|}
\hline \multirow[t]{5}{*}{ Characteristics } & \multicolumn{2}{|l|}{1990} & \multicolumn{2}{|l|}{2017} & \multicolumn{2}{|l|}{$1990-2017$} \\
\hline & $\begin{array}{l}\text { DALY No. } \times 10^{\wedge} 3 \\
(95 \% \text { UI) }\end{array}$ & $\begin{array}{l}\text { Age } \\
\text { Standardized }\end{array}$ & $\begin{array}{l}\text { DALY No. } \times 10^{\wedge} 3 \\
(95 \% \text { UI) }\end{array}$ & $\begin{array}{l}\text { Age } \\
\text { Standardized }\end{array}$ & $\begin{array}{l}\text { Change in } \\
\text { DALY(\%) }\end{array}$ & $\begin{array}{l}\text { EAPC No. } \\
(95 \% \mathrm{Cl})\end{array}$ \\
\hline & & DALY Rate & & DALY Rate & & \\
\hline & & (per 100,000$)$ & & (per 100,000$)$ & & \\
\hline & & No. $(95 \% \mathrm{UI})$ & & No. (95\% UI) & & \\
\hline Global & $\begin{array}{l}2767.67(2354.54- \\
3153.32)\end{array}$ & $\begin{array}{l}57.32(49.72- \\
64.50)\end{array}$ & $\begin{array}{l}4695.29(4433.74- \\
5186.24)\end{array}$ & $\begin{array}{l}60.66(57.21- \\
67.20)\end{array}$ & 69.65 & $\begin{array}{l}0.06(-0.05 \\
\text { to } 0.16)\end{array}$ \\
\hline \multicolumn{7}{|l|}{ Sex } \\
\hline male & $\begin{array}{l}1352.67(1134.41- \\
1558.91)\end{array}$ & $\begin{array}{l}58.50(50.53- \\
66.26)\end{array}$ & $\begin{array}{l}2120.73(1969.65- \\
2408.52)\end{array}$ & $\begin{array}{l}57.79(53.82- \\
65.44)\end{array}$ & 56.90 & $\begin{array}{l}-0.15(-0.23 \\
\text { to }-0.06)\end{array}$ \\
\hline female & $\begin{array}{l}1415.99(1185.86- \\
1645.99)\end{array}$ & $\begin{array}{l}57.14(48.81- \\
65.46)\end{array}$ & $\begin{array}{l}2574.56(2414.93- \\
2828.08)\end{array}$ & $\begin{array}{l}63.78(59.50- \\
70.46)\end{array}$ & 81.82 & $\begin{array}{l}0.21(0.08- \\
0.35)\end{array}$ \\
\hline \multicolumn{7}{|c|}{ Socio-demographic index } \\
\hline Low SDI & $\begin{array}{l}566.42(362.61- \\
807.51)\end{array}$ & $\begin{array}{l}100.67(73.39- \\
138.79)\end{array}$ & $\begin{array}{l}\text { 942.84(792.49- } \\
1191.45)\end{array}$ & $\begin{array}{l}102.52(89.14- \\
127.17)\end{array}$ & 66.46 & $\begin{array}{l}-0.08(-0.28 \\
\text { to } 0.13)\end{array}$ \\
\hline Low-middle SDI & $\begin{array}{l}688.41(557.54- \\
807.74)\end{array}$ & $\begin{array}{l}79.11(66.15- \\
91.26)\end{array}$ & $\begin{array}{l}\text { 1305.78(1198.63- } \\
1470.19)\end{array}$ & $\begin{array}{l}94.36(86.62- \\
105.72)\end{array}$ & 89.68 & $\begin{array}{l}0.54(0.44- \\
0.64)\end{array}$ \\
\hline Middle SDI & $\begin{array}{l}654.69(562.89- \\
713.74)\end{array}$ & $\begin{array}{l}50.3(43.66- \\
54.62)\end{array}$ & $\begin{array}{l}1144.02(1086.26- \\
1211.05)\end{array}$ & $\begin{array}{l}53.58(50.97- \\
56.79)\end{array}$ & 74.74 & $\begin{array}{l}0.04(-0.1 \text { to } \\
0.19)\end{array}$ \\
\hline High-middle SDI & $\begin{array}{l}487.66(444.63- \\
519.84)\end{array}$ & $\begin{array}{l}48.04(43.87- \\
51.12)\end{array}$ & $\begin{array}{l}\text { 641.04(612.9- } \\
675.45)\end{array}$ & $\begin{array}{l}39.01(37.22- \\
41.11)\end{array}$ & 31.45 & $\begin{array}{l}-1.26(-1.52 \\
\text { to }-1.01)\end{array}$ \\
\hline High SDI & $\begin{array}{l}363.11(348.24- \\
386.16)\end{array}$ & $\begin{array}{l}29.82(28.44- \\
31.85)\end{array}$ & $\begin{array}{l}655.04(627.9- \\
677.36)\end{array}$ & $\begin{array}{l}31.04(29.48- \\
32.54)\end{array}$ & 80.40 & $\begin{array}{l}0.41(0.31- \\
0.51)\end{array}$ \\
\hline \multicolumn{7}{|l|}{ Region } \\
\hline $\begin{array}{l}\text { Andean Latin } \\
\text { America }\end{array}$ & $\begin{array}{l}39.99(35.37- \\
45.20)\end{array}$ & $\begin{array}{l}\text { 101.22(92.22- } \\
111.36)\end{array}$ & $\begin{array}{l}39.97(36.07- \\
44.19)\end{array}$ & $\begin{array}{l}\text { 69.87(63.21- } \\
77.06)\end{array}$ & -0.06 & $\begin{array}{l}-1.33(-1.56 \\
\text { to }-1.11)\end{array}$ \\
\hline Australasia & $5.49(5.10-6.05)$ & $\begin{array}{l}24.05(22.23- \\
26.61)\end{array}$ & $\begin{array}{l}10.74(9.68- \\
11.83)\end{array}$ & $\begin{array}{l}23.11(20.58- \\
25.71)\end{array}$ & 95.59 & $\begin{array}{l}-0.03(-0.26 \\
\text { to } 0.20)\end{array}$ \\
\hline Caribbean & $\begin{array}{l}\text { 16.94(13.79- } \\
20.53)\end{array}$ & $\begin{array}{l}49.02(41.35- \\
57.92)\end{array}$ & $\begin{array}{l}28.58(25.85- \\
32.34)\end{array}$ & $\begin{array}{l}60.96(54.45- \\
70.26)\end{array}$ & 68.66 & $\begin{array}{l}1.10(1.02- \\
1.18)\end{array}$ \\
\hline Central Asia & $\begin{array}{l}57.67(54.98- \\
59.92)\end{array}$ & $\begin{array}{l}93.22(88.5- \\
96.88)\end{array}$ & $\begin{array}{l}107.6(101.96- \\
113.65)\end{array}$ & $\begin{array}{l}\text { 122.98(116.81- } \\
129.56)\end{array}$ & 86.56 & $\begin{array}{l}0.76(0.49- \\
1.03)\end{array}$ \\
\hline Central Europe & $\begin{array}{l}86.43(79.9- \\
89.92)\end{array}$ & $\begin{array}{l}59.96(55.51- \\
62.47)\end{array}$ & $\begin{array}{l}52.59(50.33- \\
55.29)\end{array}$ & $\begin{array}{l}27.59(26.18- \\
29.30)\end{array}$ & -39.15 & $\begin{array}{l}-2.81(-3.64 \\
\text { to }-1.98)\end{array}$ \\
\hline $\begin{array}{l}\text { Central Latin } \\
\text { America }\end{array}$ & $\begin{array}{l}80.58(76.83- \\
84.59)\end{array}$ & $\begin{array}{l}63.46(60.93- \\
66.96)\end{array}$ & $\begin{array}{l}\text { 185.82(175.07- } \\
193.44)\end{array}$ & $\begin{array}{l}78.21(73.63- \\
81.45)\end{array}$ & 130.6 & $\begin{array}{l}1.15(0.78- \\
1.52)\end{array}$ \\
\hline $\begin{array}{l}\text { Central Sub- } \\
\text { Saharan Africa }\end{array}$ & $\begin{array}{l}17.76(8.34- \\
36.86)\end{array}$ & $\begin{array}{l}37.31(24.22- \\
67.60)\end{array}$ & $\begin{array}{l}34.90(21.69- \\
61.51)\end{array}$ & $\begin{array}{l}38.67(27.23- \\
65.18)\end{array}$ & 96.49 & $\begin{array}{l}-0.11(-0.28 \\
\text { to } 0.06)\end{array}$ \\
\hline East Asia & $\begin{array}{l}354.60(276.08- \\
399.46)\end{array}$ & $\begin{array}{l}30.93(24.30- \\
34.75)\end{array}$ & $\begin{array}{l}241.09(222.17- \\
269.49)\end{array}$ & $\begin{array}{l}13.61(12.50- \\
15.18)\end{array}$ & -32.01 & $\begin{array}{l}-3.88(-4.24 \\
\text { to }-3.52)\end{array}$ \\
\hline Eastern Europe & $\begin{array}{l}211.29(185.61- \\
226.08)\end{array}$ & $\begin{array}{l}\text { 79.15(70.13- } \\
84.61)\end{array}$ & $\begin{array}{l}187.55(178.62- \\
195.95)\end{array}$ & $\begin{array}{l}62.66(59.54- \\
65.74)\end{array}$ & -11.24 & $\begin{array}{l}-1.79(-2.18 \\
\text { to }-1.39)\end{array}$ \\
\hline $\begin{array}{l}\text { Eastern Sub- } \\
\text { Saharan Africa }\end{array}$ & $\begin{array}{l}113.75(51.64- \\
239.60)\end{array}$ & $\begin{array}{l}83.79(48.36- \\
160.46)\end{array}$ & $\begin{array}{l}198.28(129.83- \\
339.93)\end{array}$ & $\begin{array}{l}81.88(55.58- \\
136.18)\end{array}$ & 74.31 & $\begin{array}{l}-0.37(-0.52 \\
\text { to }-0.22)\end{array}$ \\
\hline $\begin{array}{l}\text { High-income Asia } \\
\text { Pacific }\end{array}$ & $\begin{array}{l}32.68(30.00- \\
35.76)\end{array}$ & $\begin{array}{l}18.17(16.53- \\
20.16)\end{array}$ & $\begin{array}{l}72.37(68.01- \\
78.00)\end{array}$ & $\begin{array}{l}18.34(16.68- \\
20.29)\end{array}$ & 121.42 & $\begin{array}{l}0.36(0.17- \\
0.54)\end{array}$ \\
\hline
\end{tabular}




\begin{tabular}{|c|c|c|c|c|c|c|}
\hline $\begin{array}{l}\text { High-income } \\
\text { North America }\end{array}$ & $\begin{array}{l}143.51(137.47- \\
150.18)\end{array}$ & $\begin{array}{l}40.44(38.63- \\
42.48)\end{array}$ & $\begin{array}{l}256.83(244.63- \\
266.88)\end{array}$ & $\begin{array}{l}44.81(42.27- \\
47.01)\end{array}$ & 78.97 & $\begin{array}{l}0.41(0.21- \\
0.62)\end{array}$ \\
\hline $\begin{array}{l}\text { North Africa and } \\
\text { Middle East }\end{array}$ & $\begin{array}{l}75.44(57.87- \\
91.60)\end{array}$ & $\begin{array}{l}24.13(19.73- \\
28.11)\end{array}$ & $\begin{array}{l}113.43(105.76- \\
121.24)\end{array}$ & $\begin{array}{l}23.02(21.6- \\
24.36)\end{array}$ & 50.36 & $\begin{array}{l}-0.36(-0.51 \\
\text { to }-0.20)\end{array}$ \\
\hline Oceania & $5.88(4.89-7.20)$ & $\begin{array}{l}107.99(92.83- \\
127.47)\end{array}$ & $9.64(8.07-11.63)$ & $\begin{array}{l}93.75(81.58- \\
108.79)\end{array}$ & 63.89 & $\begin{array}{l}-0.61(-0.67 \\
\text { to }-0.55)\end{array}$ \\
\hline South Asia & $\begin{array}{l}862.39(638.05- \\
1059.68)\end{array}$ & $\begin{array}{l}97.78(75.14- \\
116.71)\end{array}$ & $\begin{array}{l}\text { 1736.91(1587.86- } \\
1917)\end{array}$ & $\begin{array}{l}117.57(107.57- \\
129.59)\end{array}$ & 101.41 & $\begin{array}{l}0.63(0.50- \\
0.76)\end{array}$ \\
\hline Southeast Asia & $\begin{array}{l}203.49(162.92- \\
231.35)\end{array}$ & $\begin{array}{l}59.57(48.73- \\
67.02)\end{array}$ & $\begin{array}{l}474.49(433.08- \\
530.05)\end{array}$ & $\begin{array}{l}77.12(70.06- \\
87.5)\end{array}$ & 133.17 & $\begin{array}{l}0.78(0.58 \\
\text { to } 0.98)\end{array}$ \\
\hline $\begin{array}{l}\text { Southern Latin } \\
\text { America }\end{array}$ & $\begin{array}{l}31.61(30.24- \\
33.25)\end{array}$ & $\begin{array}{l}66.94(64.15- \\
70.33)\end{array}$ & $\begin{array}{l}71.57(66.15- \\
77.48)\end{array}$ & $\begin{array}{l}89.23(82.49- \\
96.47)\end{array}$ & 126.40 & $\begin{array}{l}1.28(1.05- \\
1.52)\end{array}$ \\
\hline $\begin{array}{l}\text { Southern Sub- } \\
\text { Saharan Africa }\end{array}$ & $\begin{array}{l}16.03(12.42- \\
18.18)\end{array}$ & $\begin{array}{l}39.06(30.21- \\
44.41)\end{array}$ & $\begin{array}{l}29.77(26.14- \\
36.11)\end{array}$ & $\begin{array}{l}44.37(39.1- \\
53.79)\end{array}$ & 85.69 & $\begin{array}{l}0.42(-0.54 \\
\text { to } 1.39)\end{array}$ \\
\hline $\begin{array}{l}\text { Tropical Latin } \\
\text { America }\end{array}$ & $\begin{array}{l}\text { 107.54(101.47- } \\
115.2)\end{array}$ & $\begin{array}{l}87.73(83.43- \\
92.52)\end{array}$ & $\begin{array}{l}308.39(287.38- \\
320.58)\end{array}$ & $\begin{array}{l}139.82(130.67- \\
145.16)\end{array}$ & 186.76 & $\begin{array}{l}2.04(1.78- \\
2.30)\end{array}$ \\
\hline Western Europe & $\begin{array}{l}119.1(112.19- \\
131.89)\end{array}$ & $\begin{array}{l}21.31(19.92- \\
23.73)\end{array}$ & $\begin{array}{l}256.11(242.86- \\
266.10)\end{array}$ & $\begin{array}{l}27.53(25.93- \\
28.85)\end{array}$ & 115.04 & $\begin{array}{l}1.51(1.21- \\
1.81)\end{array}$ \\
\hline $\begin{array}{l}\text { Western Sub- } \\
\text { Saharan Africa }\end{array}$ & $\begin{array}{l}185.48(142.55- \\
241.57)\end{array}$ & $\begin{array}{l}\text { 105.3(85.72- } \\
128.09)\end{array}$ & $\begin{array}{l}278.68(223.69- \\
357.27)\end{array}$ & $\begin{array}{l}80.83(65.98- \\
103.68)\end{array}$ & 50.25 & $\begin{array}{l}-1.58(-1.88 \\
\text { to }-1.27)\end{array}$ \\
\hline
\end{tabular}

DALY: disability adjusted life-year; EAPC: estimated annual percentage change; Cl: confidence interval; Ul: uncertainty interval; SDI: socio-demographic index

The ASR of DALYs decreased largest in East Asia (EAPC $=-3.88,95 \% \mathrm{Cl}:-4.42$ to -3.53 ), and the increased largest in Tropical Latin America (EAPC $=+2.04,95 \% \mathrm{Cl}: 1.78$ to 2.3 ) (Fig. 2C). The ASR of DALYs only decreased in the high-middle SDI quintile, from 48.04 (95\% Ul: 43.87 to 51.12 ) to 39.01 (95\% Ul: 37.22 to 41.11 ) per 100,000 persons with an EAPC of -1.26 (95\%Cl: -1.52 to -1.01 ); the highest increase was in the low-middle SDI quintile from 79.11 (95\% Ul: 66.15 to 91.26 ) to 94.36 (95\% Ul: 86.62 to 105.72), with an EAPC of +0.54 (95\% Cl: 0.44 to 0.64 ) (Fig. 1C). DALYs decreased in other 162 countries, the United Arab Emirates increased largest (+ 835.04\%); the ASR of DALYs was highest in Tajikistan (253.89 per 100,000 persons), and increased largest in Portugal (EAPC $=+$ 6.68, 95\% Cl: 5.79 to 7.58 ) (Supplementary Table 4 and Fig. 7).

ASR of DALYs mostly showed a decrease trend with the SDI, but only increased at SDI values from 0.60 to 0.68 (Fig. 4C). EAPC was negatively correlated with ASR of DALYS $(\rho=-0.38, p<.001)$, but not correlated with SDI $(\rho=0.08, p=.28$, Fig. 5C).

\section{Age Distribution of Incidence, Deaths and DALYs of Urinary Tract Infections}

From 1990 to 2017, incident cases of UTIs mainly concentrated in the 15-49 age group (Fig. 8A). The largest number of deaths were in the age group above 70. It is noteworthy that the proportion of deaths in the under 5, 5-14, and 15-49 age groups decreased year by year (Fig. 8B). The proportion of DALYs decreased year by year in under 5 age group, while increased in over 70 age group (Fig. 8C).

Both in 1990 and 2017, the incidence rate (per 100,000 persons) of UTIs in males and females showed different trends in each age group. Incidence rates were highest in 30-34 age group for females and over 80 age group for males (Fig. 9A). The death rates were highest in over 80 age group for female and males (Fig. 9B), and DALYs rate was highest in age group of under 1 and over 80 for females and males (Fig. 9C).

\section{Discussion}

We analyzed the burden of disease due to UTIs for 195 countries and territories, 21 regions, and 5 SDI quintiles from 1990 to 2017. Globally, the incident cases, death cases, and DALYs of UTIs increased by $52.09 \%, 140.10 \%$, and $69.65 \%$, respectively. The ASIR, ASDR, and ASR of DALYs all showed an upward trend. The highest ASIR was observed in New Zealand and Norway. The highest ASDR was observed in Seychelles and Barbados. These four countries showed a heavy disease burden of UTIs and indicates that 
disease-related preventive measures and treatment methods need to be improved. India and China have the highest number of incident cases in 2017. However, the ASIR showed a decrease in China, which may be related to the huge population base, aging and expansion of medical care coverage.

Our results also showed that ASIR was highest in the high SDI quintile and lowest in the low SDI quintile, and showed a positive correlation with SDI. To some extent, living habits and environment may play a role. More importantly, there may be numerous patients with UTIs who did not choose regular medical treatments or some areas do not have medical conditions to provide the correct diagnosis and record it. On the contrary, ASDR were highest in low SDI quintile and lowest in high-middle SDI quintile. This indicates that areas with relatively high SDI values may have superior medical resources, and it also proves the hypothesis that areas with low SDI values may not provide sufficient diagnostic and treatment resources. Therefore, these data from countries and regions with insufficient medical conditions, which may mention us should be put more attention and analyses.

In 2017, the incidence cases of UTIs in females were 3.44 times higher than males, the deaths cases were 1.31 times higher, and DALYs was 1.21 times higher. These results were consistent with previous published studies, which have found there was approximately $30-44 \%$ of females would have a recurrent UTIs within six months of an initial infection $(12,13)$. Obviously, recurrent UTIs incur a huge economic burden each year due to prevention and treatment (14). The high incidence and recurrence rate of females may relate to many factors, such as physiological structure of the female urinary tract, changes in the acidic environment of the vagina caused by age-related decline in estrogen levels, genetic factors and pregnancy status(15). In a word, the formulation of preventive strategies for female patients is of practical significance for reducing the burden of disease caused by UTIS.

Infants, who is younger than 1 year old, have a higher incidence rate of UTIs, and females were higher than males of them in 2017 ( $2.88 \%$ vs. $1.63 \%)$. Other studies also reported over $30 \%$ of infants and children experience recurrent infections within 6-12 months after the initial infection $(16,17)$, and $10 \%-40 \%$ children with febrile UTIs have permanent renal scarring $(18,19)$. The disease burden of UTIs in infants and children may be reduced by male circumcision(20), maintain perineal hygiene(21), treatment for lactating women(22). Female between 25-35 years old showed a high incidence rate of UTIs, which is also the peak period of female pregnancy. Moreover, Masinde's study showed an estimated $17.9 \%$ and $13.0 \%$ of pregnant women had symptomatic UTIs and ASB, respectively(23). As we know, UTIs during pregnancy can have adverse outcomes for both pregnant women and fetuses. Hence, rational use of antibiotics not only reduces the disease burden of urinary tract infections, but also reduces antibiotic resistance and drug side effects.

Although GBD 2017 provide comprehensive data about the global disease burden of UTIs, it still has some limitations. First, the data has not yet covered all regions of the world, relevant studies in these regions remain needed, also may provide methodological help for them. Second, since there were differences in the diagnostic methods in different regions, the data may not be very accurately reflecting the burden of UTIs in individual areas, and we suggest to unify the diagnostic methods and training before perform in future investigation. Third, current GBD study lack of specific classification data such as complex and non-complexity UTIs, and specific groups such as pregnant women and hospitalized elder. Hence, we suggest adding more disease-related information in the GBD database to facilitate a more comprehensive assessment of the burden of disease caused by UTIs.

\section{Conclusion}

The disease burden of UTIs increased from 1990 to 2017 globally, especially in females; however, distinct varies were observed in different regions and countries. The infants and elders are easier to die when they suffer from UTIs.

\section{Abbreviations}

\section{UTIs}

Urinary tract infections

DALYs

Disability adjusted life-years

EAPC

Estimated annual percentage changes

ASIR 
Age-standardized incidence rate

ASDR

Age-standardized death rate

SDI

Socio-demographic index

GBD 2017

Global Burden of Disease Study 2017

ASR

Age standardized rates

$95 \%$ UI

95\% uncertainty interval

ICD

The International Classification of Diseases

\section{Declarations}

\section{Ethics approvaland consent to participate}

This article does not contain any studies with human participants or animals performed by any of the authors. The GBD study was approved by the Institutional Review Board of the University of Washington. As no individual patient data was collected, consent to participate does not apply.

\section{Consent to publication}

Not applicable.

\section{Competing interests}

The authors declare that they have no competing interests.

\section{Availability of data and materials}

The datasets used and/or analyzed during the current study are available from the corresponding author on reasonable request. Data we used in this article are publicly available online on the official website of Institute of Health Metrics and Evaluation (http://ghdx.healthdata.org/gbd-results-tool).

\section{Funding}

This work was supported by the Nature Science Foundation of Hubei Province (2019CFB760), the Health Commission of Hubei Province Scientific Research Project (WJ2019H035), and the National Key Research and Development Plan of China (2016YFC0106302). The funder had no role in study design, data collection and analysis, decision to publish, or preparation of the manuscript. The authors declare that there are no conflicts of interest in this study.

\section{Author's Contribution}

CZ: Date collection, Data analysis, Manuscript writing

QH and LL: Data analysis

TD, JG, ST and LL: Data collection

$\mathrm{XZ}$ and HZ: Designed the study, Supervised the research and Reviewed the manuscript

\section{Acknowledgements}

We are grateful to the work by the Global Burden of Disease Study 2017 collaborators. 


\section{References}

1. Detweiler K, Mayers D, Fletcher SG. Bacteruria and Urinary Tract Infections in the Elderly. The Urologic clinics of North America. 2015;42(4):561-8.

2. Foxman B, Barlow R, D'Arcy H, Gillespie B, Sobel JD. Urinary tract infection: self-reported incidence and associated costs. Annals of epidemiology. 2000;10(8):509-15.

3. McLellan LK, Hunstad DA. Urinary Tract Infection: Pathogenesis and Outlook. Trends in molecular medicine. 2016;22(11):946-57.

4. Shaikh N, Morone NE, Bost JE, Farrell MH. Prevalence of urinary tract infection in childhood: a meta-analysis. The Pediatric infectious disease journal. 2008;27(4):302-8.

5. Bonadio W, Maida G. Urinary tract infection in outpatient febrile infants younger than 30 days of age: a 10-year evaluation. The Pediatric infectious disease journal. 2014;33(4):342-4.

6. Ahmed H, Farewell D, Jones HM, Francis NA, Paranjothy S, Butler CC. Incidence and antibiotic prescribing for clinically diagnosed urinary tract infection in older adults in UK primary care, 2004-2014. PloS one. 2018;13(1):e0190521.

7. Foxman B. Epidemiology of urinary tract infections: incidence, morbidity, and economic costs. Disease-a-month : DM. 2003;49(2):53-70.

8. Simmering JE, Tang F, Cavanaugh JE, Polgreen LA, Polgreen PM. The Increase in Hospitalizations for Urinary Tract Infections and the Associated Costs in the United States, 1998-2011. Open forum infectious diseases. 2017;4(1):ofw281.

9. Global, regional, and national incidence, prevalence, and years lived with disability for 354 diseases and injuries for 195 countries and territories, 1990-2017: a systematic analysis for the Global Burden of Disease Study 2017. Lancet (London, England). 2018;392(10159):1789-858.

10. Global, regional, and national comparative risk assessment of 84 behavioural, environmental and occupational, and metabolic risks or clusters of risks for 195 countries and territories, 1990-2017: a systematic analysis for the Global Burden of Disease Study 2017. Lancet (London, England). 2018;392(10159):1923-94.

11. Global, regional, and national age-sex-specific mortality and life expectancy, 1950-2017: a systematic analysis for the Global Burden of Disease Study 2017. Lancet (London, England). 2018;392(10159):1684-735.

12. Foxman B. Urinary tract infection syndromes: occurrence, recurrence, bacteriology, risk factors, and disease burden. Infectious disease clinics of North America. 2014;28(1):1-13.

13. Gupta K, Trautner BW. Diagnosis and management of recurrent urinary tract infections in non-pregnant women. BMJ (Clinical research ed). 2013;346:f3140.

14. Gaitonde S, Malik RD, Zimmern PE. Financial Burden of Recurrent Urinary Tract Infections in Women: A Time-driven Activitybased Cost Analysis. Urology. 2019;128:47-54.

15. Dielubanza EJ, Schaeffer AJ. Urinary tract infections in women. The Medical clinics of North America. 2011;95(1):27-41.

16. Nuutinen $M$, Uhari M. Recurrence and follow-up after urinary tract infection under the age of 1 year. Pediatric nephrology (Berlin, Germany). 2001;16(1):69-72.

17. Mangiarotti P, Pizzini C, Fanos V. Antibiotic prophylaxis in children with relapsing urinary tract infections: review. Journal of chemotherapy (Florence, Italy). 2000;12(2):115-23.

18. Benador D, Benador N, Slosman D, Mermillod B, Girardin E. Are younger children at highest risk of renal sequelae after pyelonephritis? Lancet (London, England). 1997;349(9044):17-9.

19. Hewitt IK, Zucchetta P, Rigon L, Maschio F, Molinari PP, Tomasi L, et al. Early treatment of acute pyelonephritis in children fails to reduce renal scarring: data from the Italian Renal Infection Study Trials. Pediatrics. 2008;122(3):486-90.

20. Morris BJ, Wiswell TE. Circumcision and lifetime risk of urinary tract infection: a systematic review and meta-analysis. The Journal of urology. 2013;189(6):2118-24.

21. Korbel L, Howell M, Spencer JD. The clinical diagnosis and management of urinary tract infections in children and adolescents. Paediatrics and international child health. 2017;37(4):273-9.

22. Santen SA, Altieri MF. Pediatric urinary tract infection. Emergency medicine clinics of North America. 2001;19(3):675-90.

23. Masinde A, Gumodoka B, Kilonzo A, Mshana SE. Prevalence of urinary tract infection among pregnant women at Bugando Medical Centre, Mwanza, Tanzania. Tanzania journal of health research. 2009;11(3):154-9.

Page $12 / 20$ 
Figures

A

both
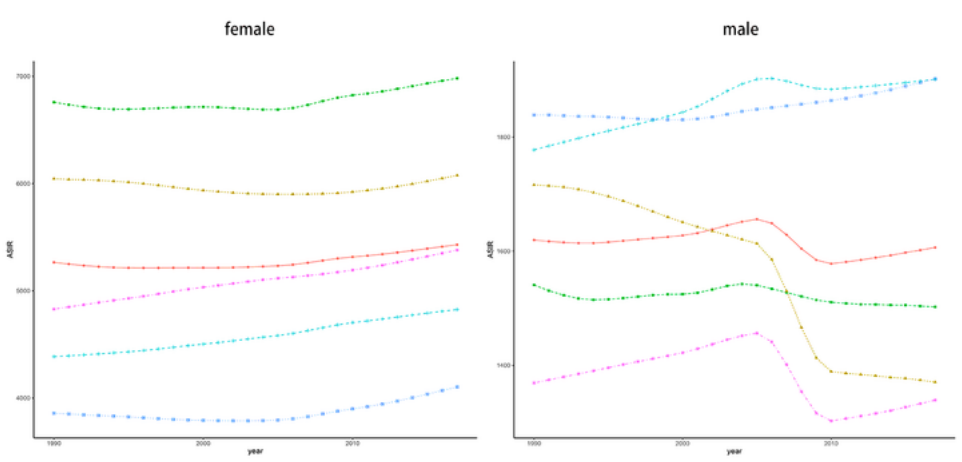

B
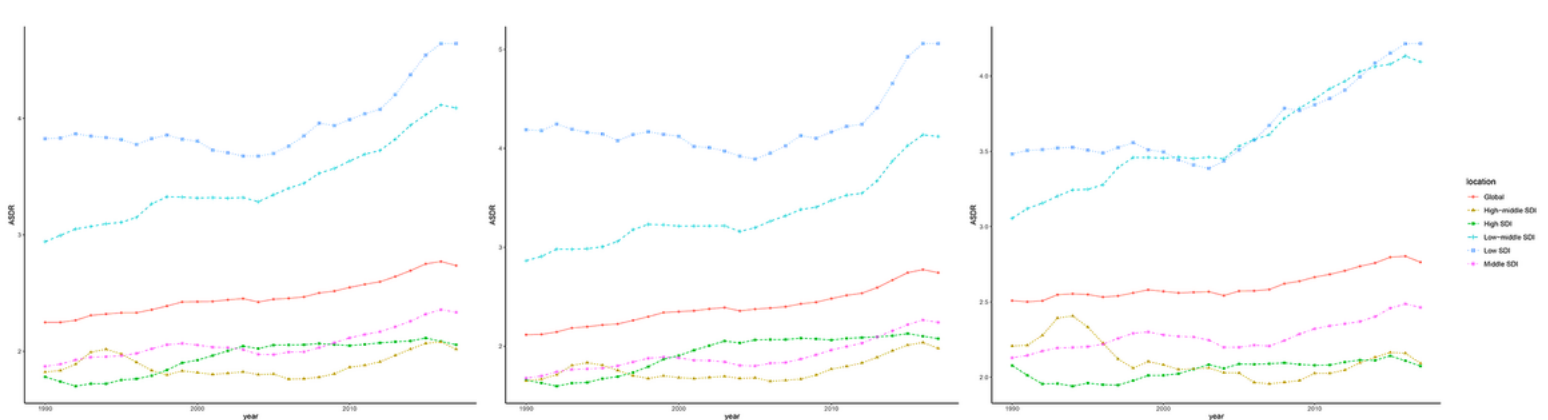

C
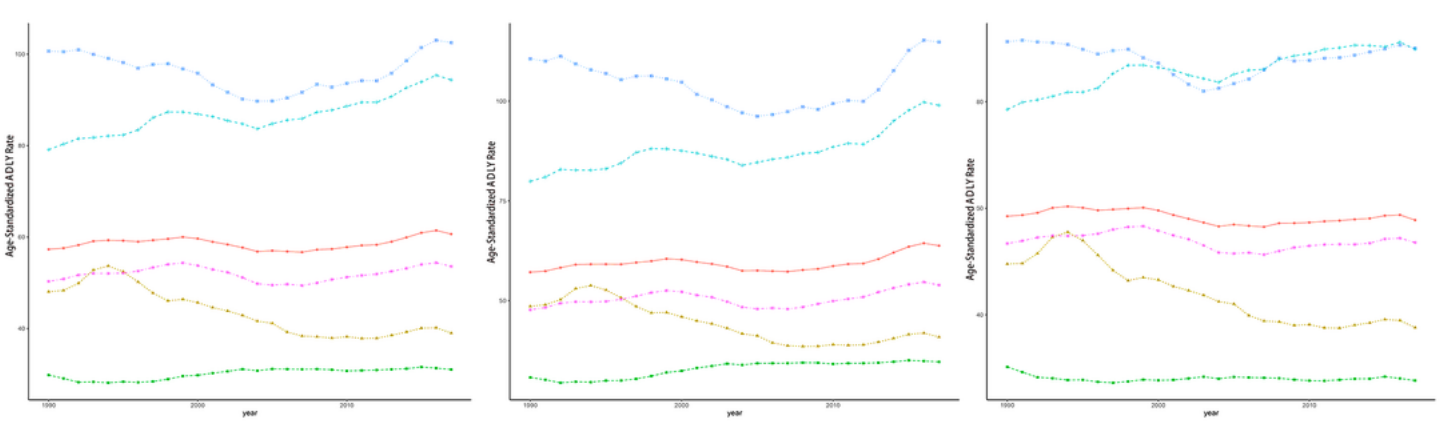

\section{Figure 1}

The change trends of age-standardized rate among different SDI quintiles and gender from 1990 to 2017. a ASIR: age-standardized incidence rate; b ASDR: age-standardized death rate; c age-standardized DALYs rate. DALYs, disability adjusted life-years; SDI, sociodemographic index 


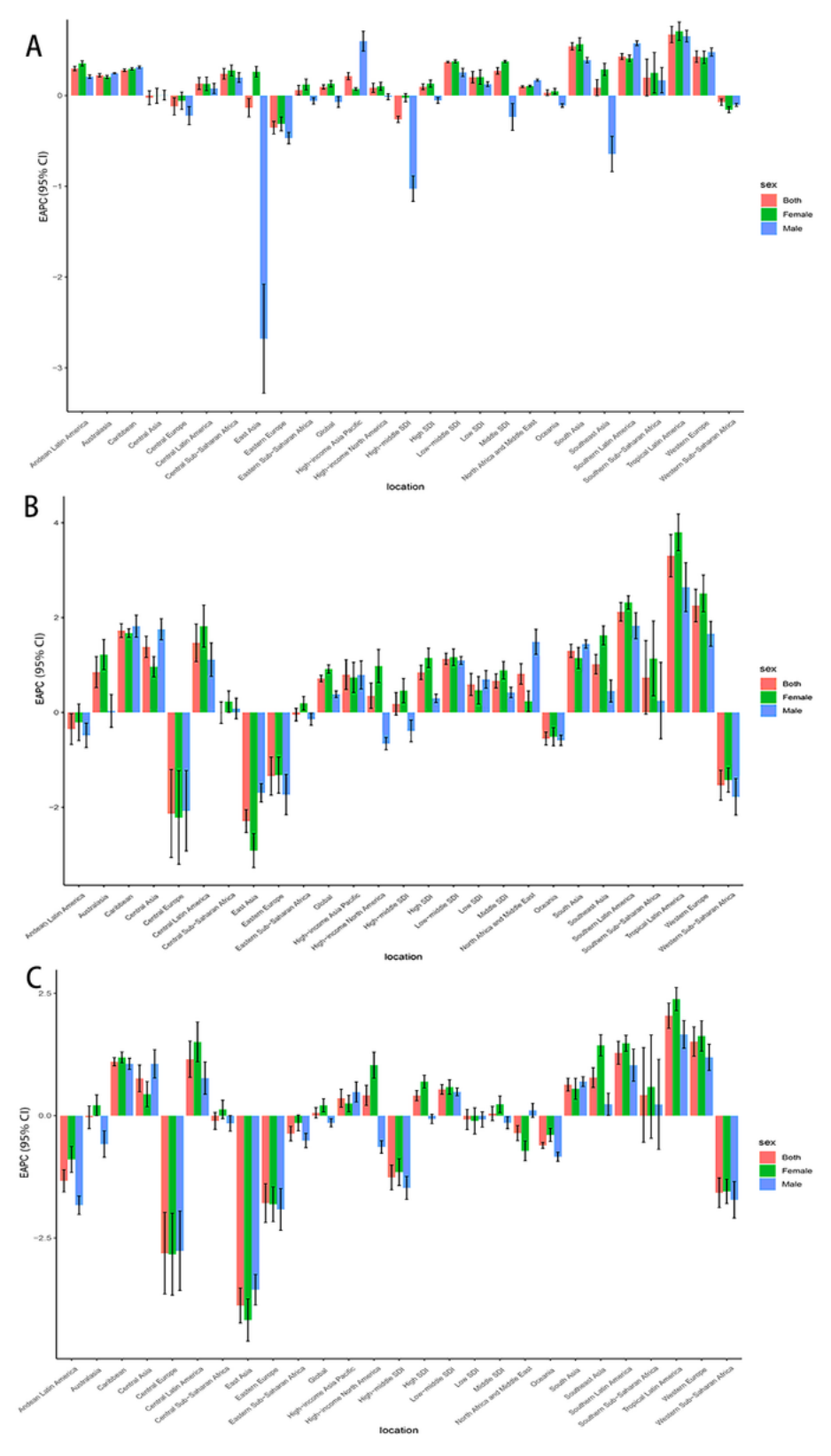

\section{Figure 2}

The EAPC of UTIs from 1990 to 2017, by sex and region. a The EAPC of ASIR; $b$ The EAPC of ASDR; c The EAPC of ASR of DALYs. ASR: age-standardized rate; ASIR: age standardized incidence rate; ASDR: age standardized death rate; EAPC, estimated annual percentage change; DALYs: disability adjusted life-years 
A
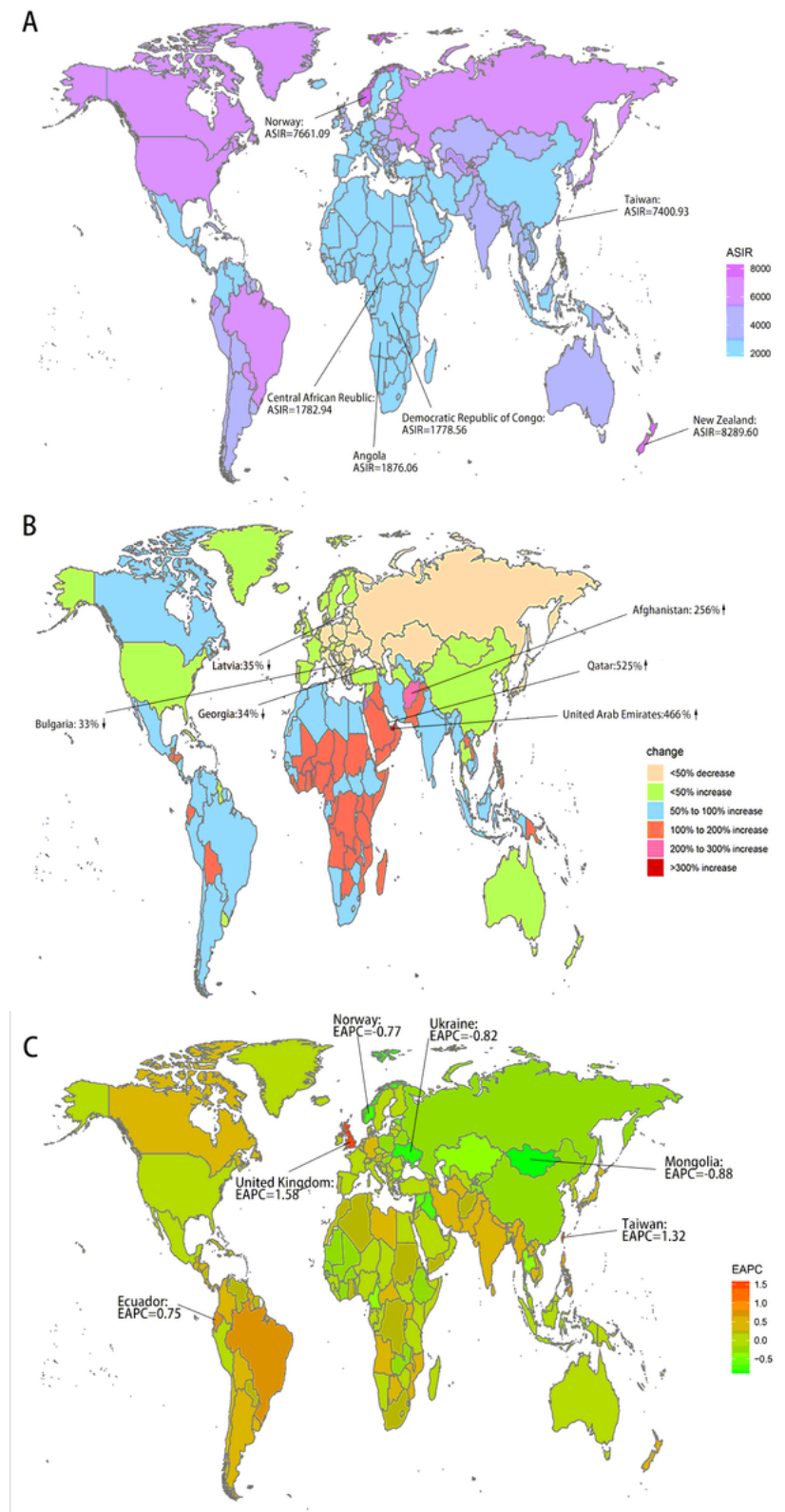

\section{Figure 3}

The global incidence burden of urinary tract infections in 195 countries and territories. a The ASIR of UTIs in 2017; b the relative change in incident cases of UTIs between 1990 and 2017; c The EAPC of UTIs from 1990 to 2017. Countries or territories with an extreme number of cases or changes were lined out. ASIR, age-standardized incidence rate; EAPC, estimated annual percentage change Note: The designations employed and the presentation of the material on this map do not imply the expression of any opinion whatsoever on the part of Research Square concerning the legal status of any country, territory, city or area or of its authorities, or concerning the delimitation of its frontiers or boundaries. This map has been provided by the authors. 

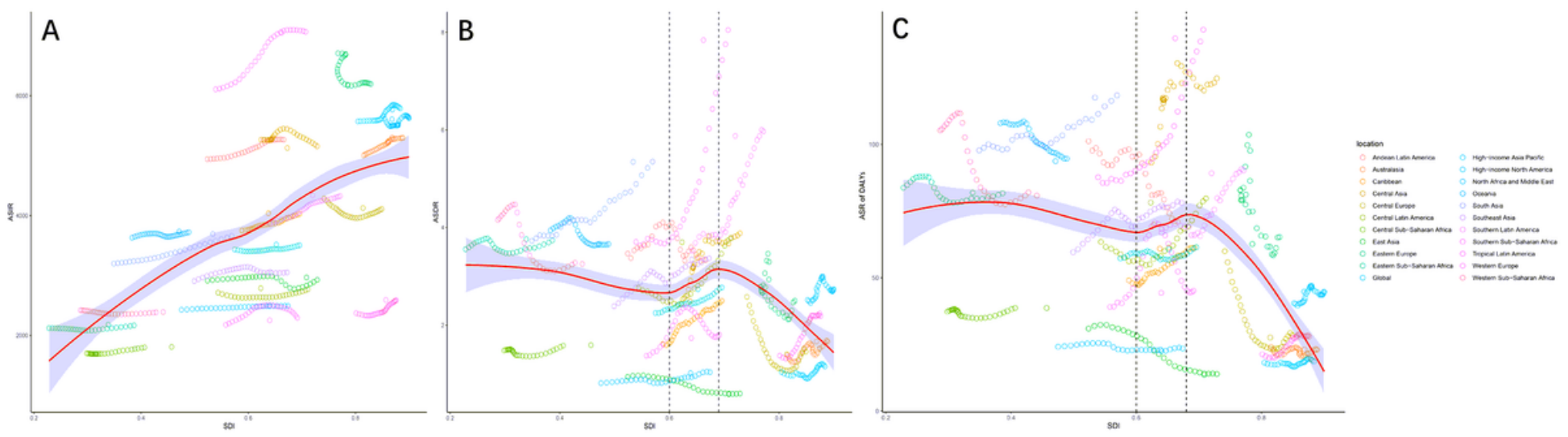

\section{Figure 4}

Correlation between the ASR and SDI in 2017 among urinary tract infections. a ASIR; b ASDR; c ASR of DALYs. ASR, age-standardized rate; ASIR, age-standardized incidence rate; ASDR, age-standardized death rate SDI, socio-demographic index, DALYs, disability adjusted life-years

A
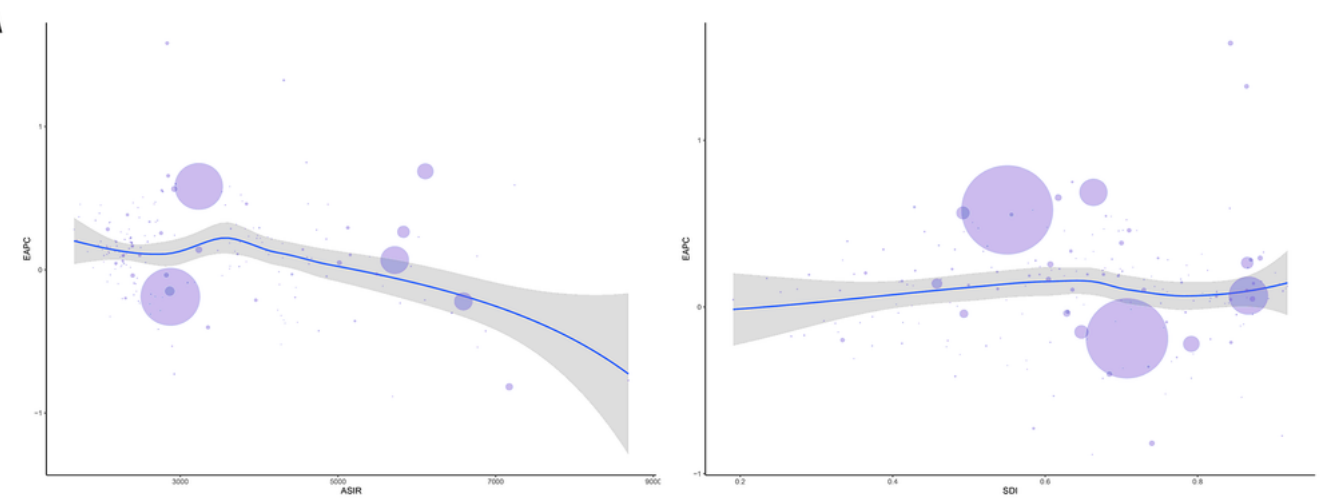

B
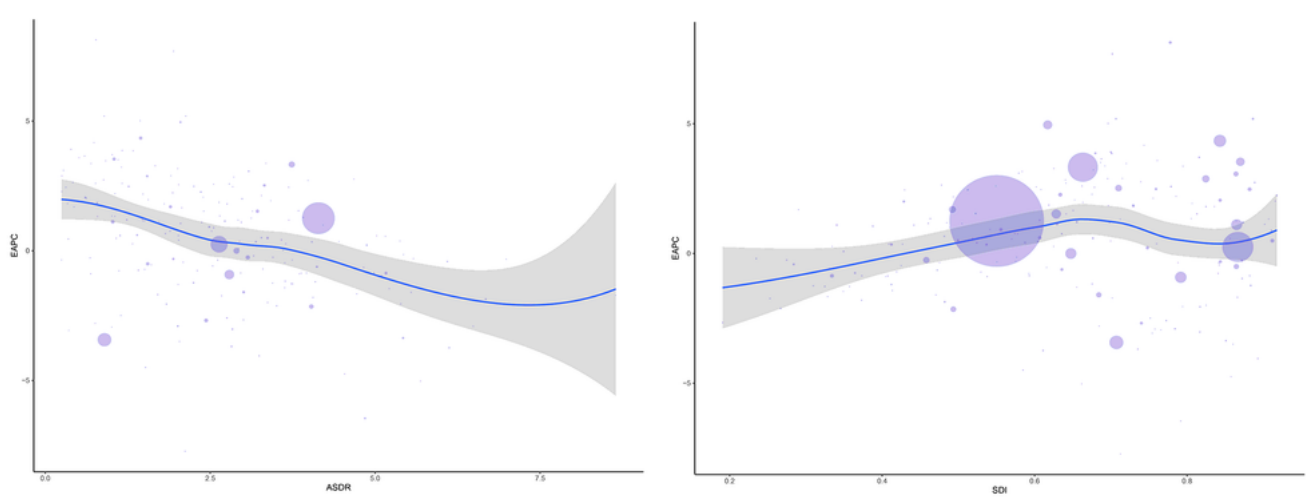

C
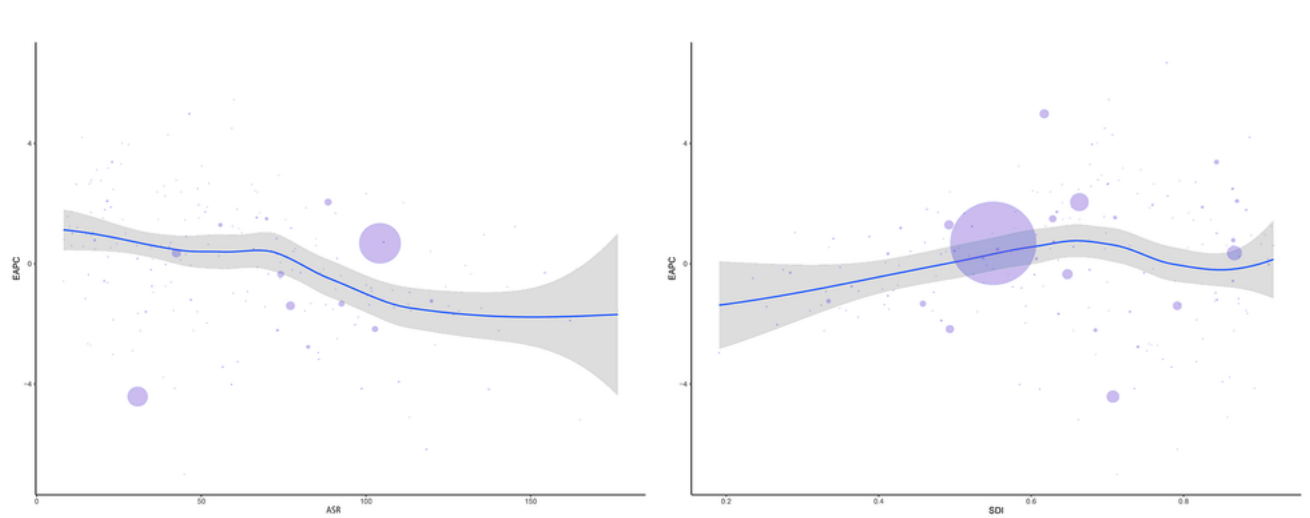

Figure 5

Page 16/20 
Correlation between the EAPC and the urinary tract infections ASR in 1990 and SDI in 2017. a incidence; b death; c DALYs. The circles represent countries that were available on SDI data. The size of circle is increased with the cases of urinary tract infections: a cases per $1,000,000$, b cases per $1,000, c$ cases per 30,000. The $\rho$ indices and $p$ values presented were derived from Pearson's correlation analysis. ASIR, age-standardized incidence rate; ASDR, age-standardized death rate; DALYs, disability adjusted life-years; EAPC, estimated annual percentage change; SDI, socio-demographic index

A
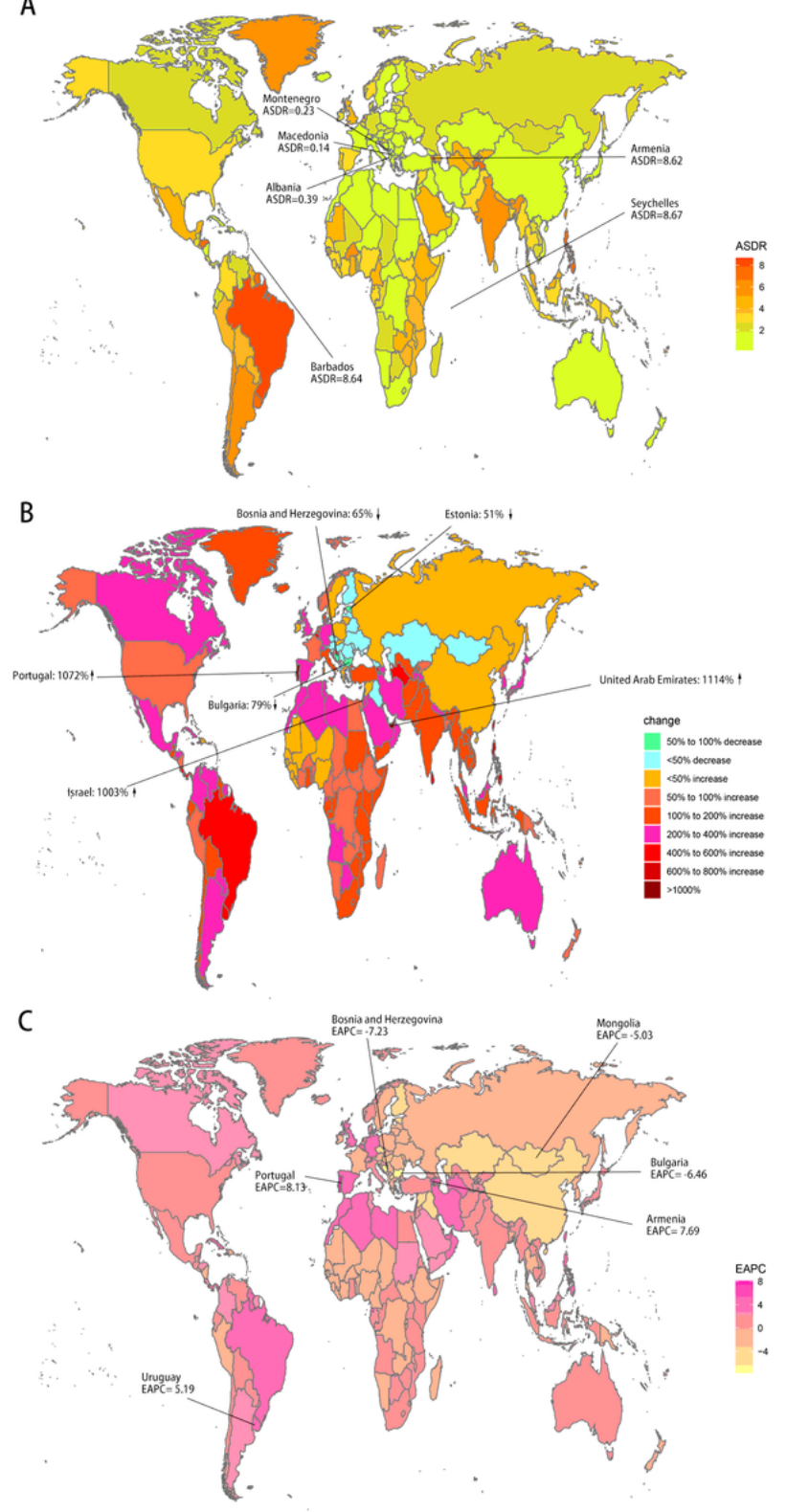

\section{Figure 6}

The global death burden of urinary tract infections in 195 countries and territories. a the ASDR of UTIs in 2017; $b$ the relative change in deaths of UTIs between 1990 and 2017; c The EAPC of UTIS ASDR from 1990 to 2017. Countries or territories with an extreme number of cases or changes were lined out. ASDR, age-standardized death rate; EAPC, estimated annual percentage change. Note: The designations employed and the presentation of the material on this map do not imply the expression of any opinion whatsoever on the part of Research Square concerning the legal status of any country, territory, city or area or of its authorities, or concerning the delimitation of its frontiers or boundaries. This map has been provided by the authors. 
A
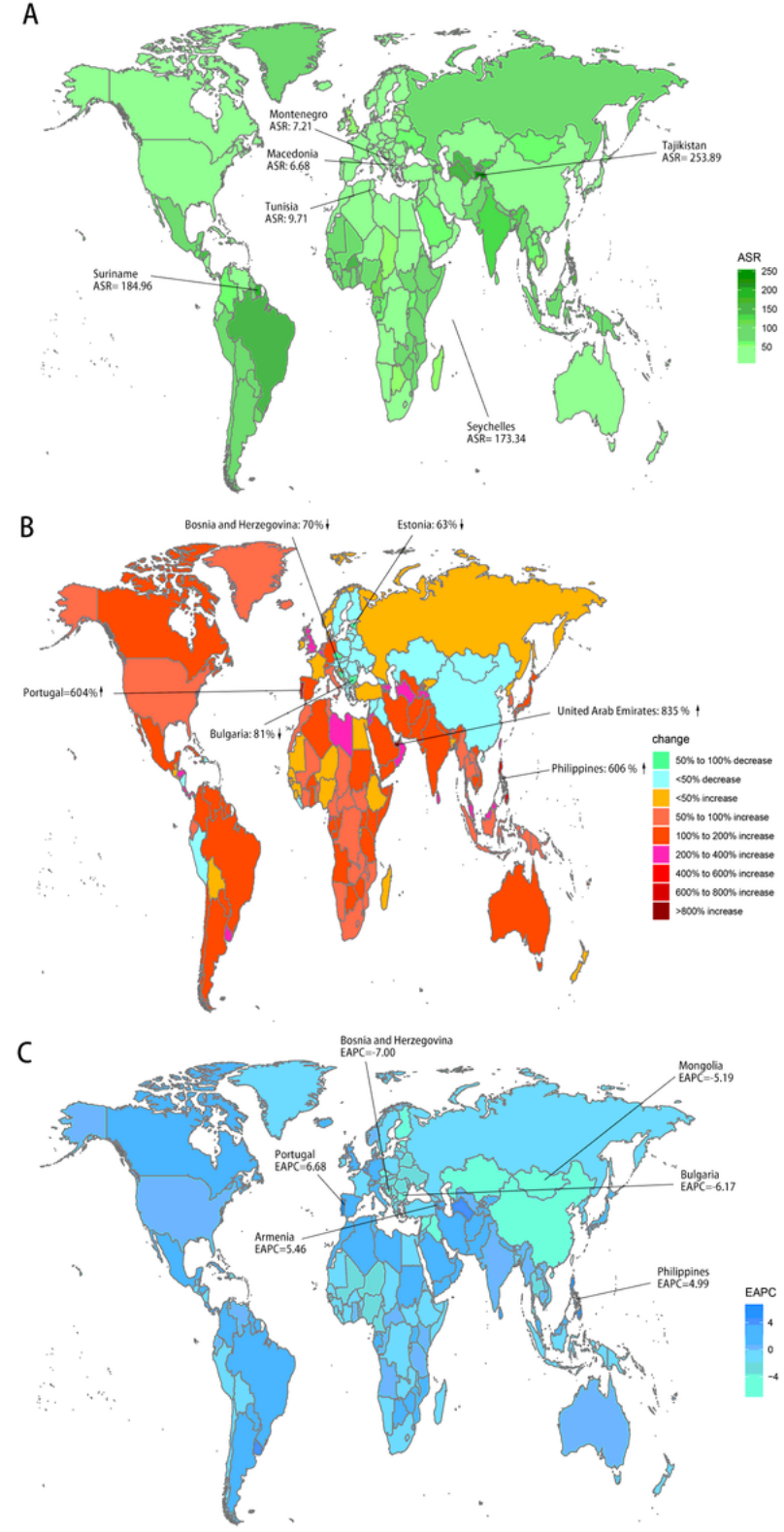

\section{Figure 7}

The global DALYs burden of urinary tract infections in 195 countries and territories. a the age-standardized DALYs rate of UTIs in 2017; b The relative change in DALYs of UTIs between 1990 and 2017; c The EAPC of UTIs age-standardized DALYs rate from 1990 to 2017. Countries or territories with an extreme number of cases or changes were lined out. DALY, disability adjusted life-year; EAPC, estimated annual percentage change. Note: The designations employed and the presentation of the material on this map do not imply the expression of any opinion whatsoever on the part of Research Square concerning the legal status of any country, territory, city or area or of its authorities, or concerning the delimitation of its frontiers or boundaries. This map has been provided by the authors. 


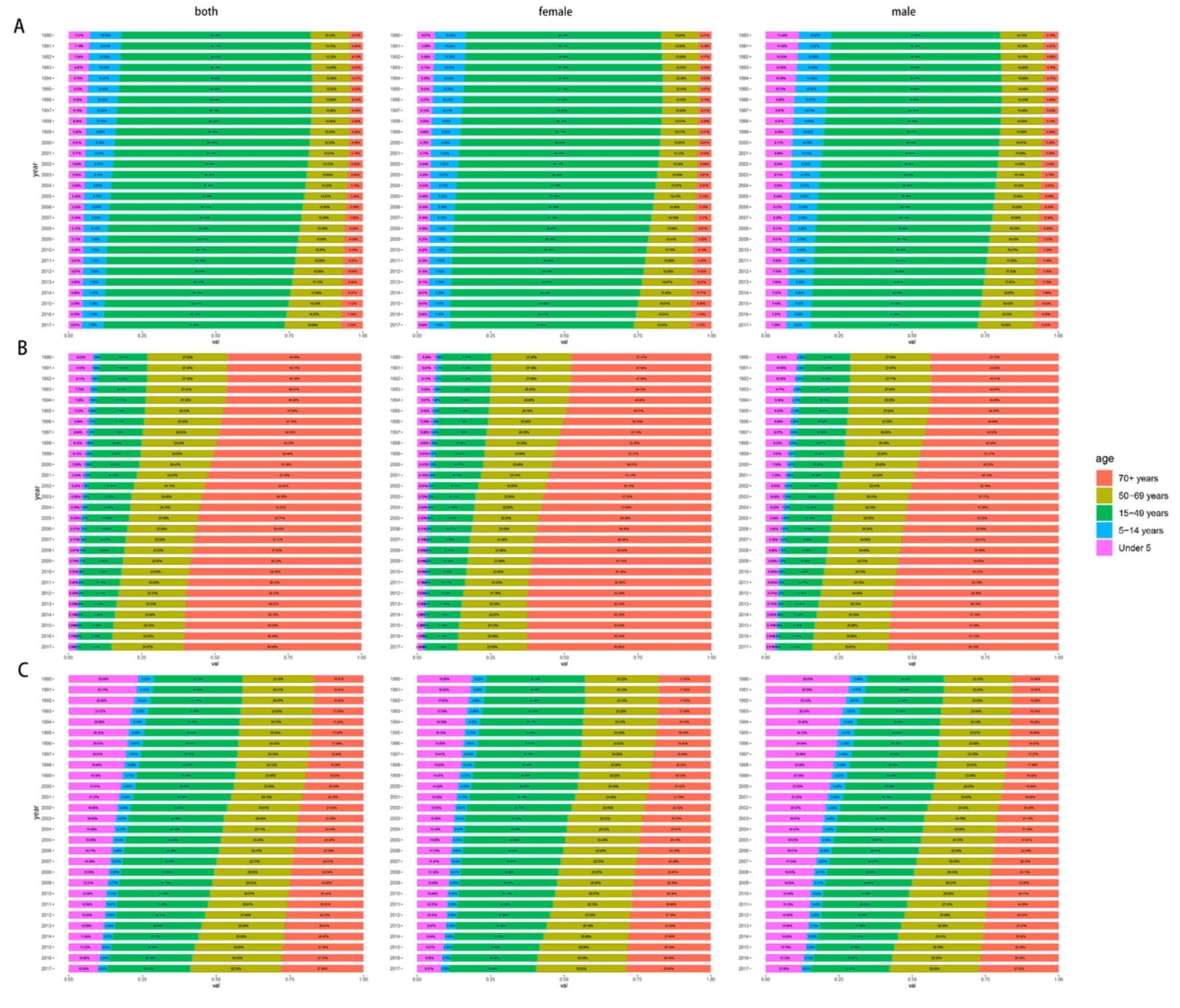

\section{Figure 8}

The proportion of different age groups in urinary tract infections from 1990 to 2017 . a incidence, b death, c DALYs. DALYs: disability adjusted life-years 
A

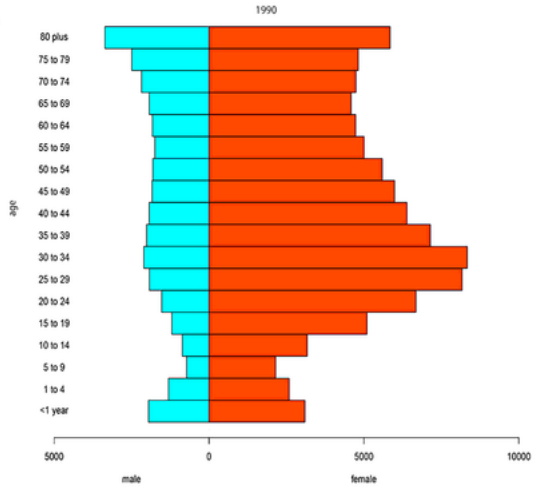

B

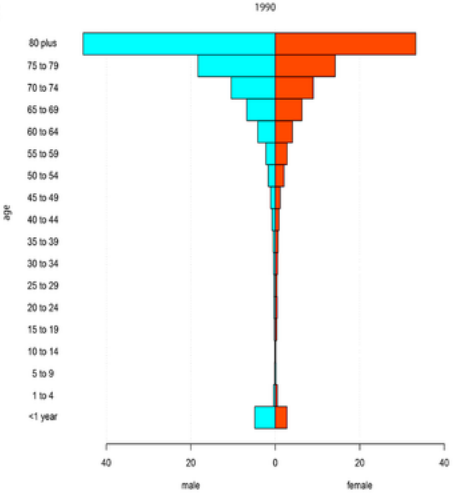

C

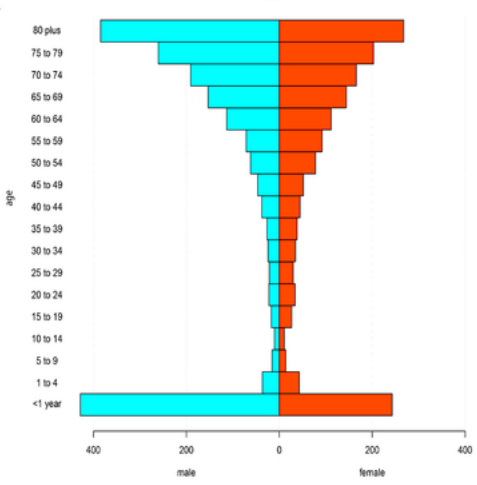

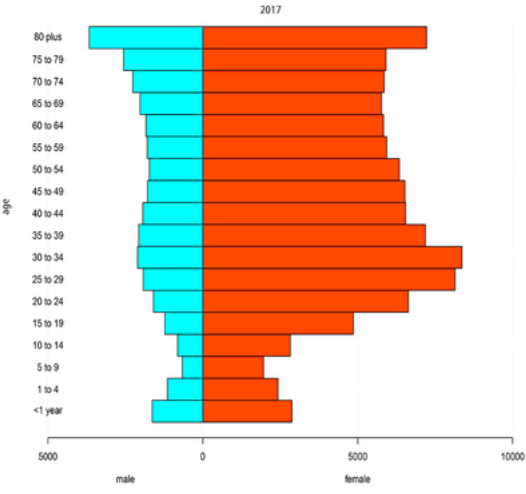

2017

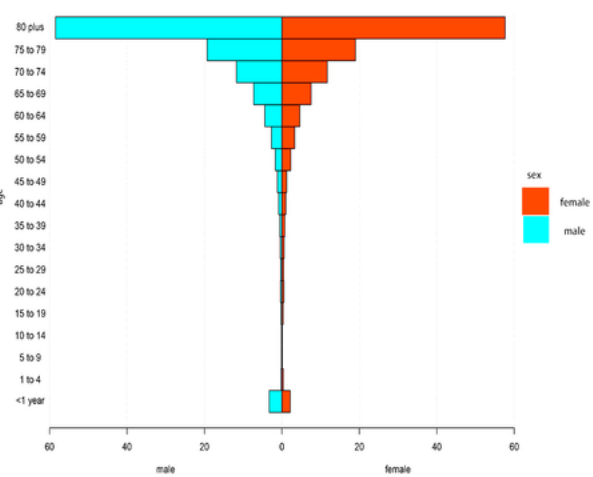

2017

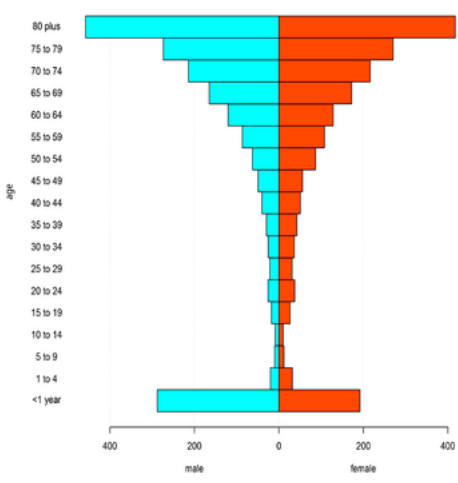

Figure 9

The rate of urinary tract infection (per 100,000 persons) among gender and age in 1990 and 2017. a incidence rate; b death rate; $c$ DALYs rate. DALYs, disability adjusted life-year

\section{Supplementary Files}

This is a list of supplementary files associated with this preprint. Click to download.

- Additionalfile.pdf 